\title{
Introducing Locally Affine-invariance Constraints into Lunar Surface Image Correspondence
}

\author{
Yu-Ren Zhang ${ }^{\mathrm{a}}$, Xu Yang ${ }^{\mathrm{a}, *}$, Hong Qiao ${ }^{\mathrm{a}}$, Zhi-Yong Liu ${ }^{\mathrm{a}}$, Chuan-Kai Liu \\ ${ }^{a}$ State Key Laboratory of Management and Control for Complex Systems, \\ Institute of Automation, Chinese Academy of Sciences, Beijing, China \\ ${ }^{b}$ Beijing Aerospace Flight Control Center, Beijing, China
}

\begin{abstract}
This paper aims to solve the keypoint correspondence problem in lunar surface images, a typical correspondence task under point ambiguity. Point ambiguity may be caused by repetitive patterns, cluttered scenes, and outliers in the images, which makes the local descriptors less discriminative. In this paper we introduce locally affine-invariance constraints on graphs to tackle the keypoint correspondence problem under point ambiguity. The key idea is that each point can be represented with the affine combination of its neighbours. It is suitable for our problem because it is not only invariant to scale and rotational change, but also more resistant to outliers. Specifically, we introduce the locally affine-invariance constraints into the subgraph matching problem and the common subgraph matching problem. The locally affine-invariance constraint is not directly applicable on common subgraph matching due to its dependency on awareness of selected keypoints. This problem is approximately addressed by solving a series of reliable matching identification and rematching problems. In the experiments, we first apply the proposed method on standard graph matching datasets to evaluate its effectiveness on general correspondence problem under point ambiguity, and second validate the applicability on the lunar surface image dataset.
\end{abstract}

Keywords: keypoint correspondence, point ambiguity, graph matching, lunar image processing

\footnotetext{
*Corresponding author.

Email addresses: yuren.zhang@ia.ac.cn (Yu-Ren Zhang), xu.yang@ia.ac.cn (Xu Yang), hong.qiao@ia.ac.cn (Hong Qiao), zhiyong.liu@ia.ac.cn (Zhi-Yong Liu), ckliu2015@126.com (Chuan-Kai Liu)

(C) 2016. This manuscript version is made available under the Elsevier user license http://www.elsevier.com/open-access/userlicense/1.0/
} 


\section{Introduction}

In many computer vision tasks, such as image stitching [1], 3D reconstruction [2] and object detection [3, 4], one of the core steps is to establish reliable correspondence of points between two images. Local descriptor based point correspondence methods may be the most popular methods, especially when equipmented with scale and rotation invariant local descriptors[5]. However, under some circumstances such as the lunar surface, there are various factors that make the point matching problem difficult. The first factor is repetitive patterns which may confuse matches. Secondly, outliers may exist in both point sets that have no corresponding points in the other. Both factors make the points ambiguous in the matching problem. Matching under point ambiguity frequently arises in planetary image analysis [6] and cluttered scenes $[7,8]$.

Matching under point ambiguity calls for constraint on the mapping. To tackle this problem, many existing point matching algorithms model keypoint matching problem as a graph matching problem and utilize geometrical constraint on the mapping. Distance and orientation are the most commonly used features for enforcing geometric consistency $[9,10,11]$. However they are sensitive to scale, orientation, and outliers [12]. To make the constraint invariant to scale and orientation change, high order affinities have been utilized. Triplets similarity is used in [13], and [14] uses a tensor-based algorithm to solve the high order correspondence problem. Unfortunately, these methods usually require higher computational and space complexity. The graph matching based scheme is first utilized to deal with the lunar surface image keypoint correspondence problem in [6]. It formulates the correspondence problem as subgraph matching problem and adopts a probabilistic graph matching algorithm. The assignment probability is ranked to find specified number of best assignments. Such a two-step scheme may be inappropriate because even both steps are optimally solved, the final matching solution may not be the optimal [15].

In this paper, to tackle the task of lunar surface keypoint correspondence, an effective constraint, i.e., locally affine-invariance constraint, is introduced into the graph matching framework. The locally affine-invariance constraint is robust against repeated patterns, affine transformation, and outliers while does not incur higher computational complexity. The optimization is NP- 
hard. It is efficiently solved with a novel path-following based algorithm.

The three main contributions are: (1) the locally affine-invariance constraint is introduced into graph based lunar surface image keypoint correspondence problem, and a novel objective function is constructed such that the similarity of corresponding points local structure is preserved and the dependency on local feature is further alleviated, (2) a novel path following algorithm is generalized to solve the optimization problem and a normalization process is proposed to regulate both the balance between pairwise and locally affine costs, and the optimization process, (3) to tackle the inapplicability of locally affine-invariance constraint on common subgraph matching problem due to the dependency on the awareness of selected nodes in the correspondence, an algorithm is proposed which iteratively identifies reliable matches and solve rematching problem with affine representation cost built upon the reliable matches.

The remainder of this paper is organized as follows. Related works are introduced in Section 2. Then the common subgraph model of the lunar surface image correspondence problem is built in Section 3. The locally affineinvariance constraint based matching algorithm is introduced in Section 4. Experiments are reported in Section 5 and Section 6 concludes the paper.

\section{Related Works}

Two groups of correspondence algorithms, appearance descriptor based correspondence and graph matching based correspondence, are reviewed below. In particular, we focus on the papers which cope with ambiguous matches and outliers.

\subsection{Appearance Descriptor based Correspondence}

Key point correspondence can be solved by using a set of local interest points. Scale-invariant feature transformation (SIFT) [5] is a frequently used appearance descriptor for key point correspondence. It has been shown to be invariant to rotation and scale, and robust to local geometrical distortion. Other popular descriptors include speeded up robust features (SURF) [16], maximally stable extremal regions (MSER) [17], binary robust invariant scalable keypoints (BRISK) [18], and etc. Though local features are often effective for many key point correspondence problems, they often fail to cope with outliers and ambiguous patterns. An improvement strategy is the NNratio decision criterion which computes the ratio between the distance to the 
closest neighbor in the feature space and the distance to the second-closest one [5]. and considers the matching to be ambiguous if the ratio of the closest distance to the second closet distance is large. The algorithm [19] improved the SIFT feature with global context to cope with the ambiguity caused by repetitive patterns. However, although these works have improved the performance of appearance descriptor, relying on local features alone is usually insufficient for key point matching in complex circumstances.

\subsection{Graph Matching based Correspondence}

Incorporating local pairwise geometrical constraints is an effective way to improve the performance of appearance descriptor based algorithms. When pairwise constraints such as distance and orientation are used, the key point correspondence problem can be formulated as a graph matching problem. However, the problem is known to be NP-hard, and therefore many approximate matching algorithms have been proposed as introduced below [20, 21].

Spectral matching is an efficient approach for graph matching. The eigendecomposition method proposed in [22] is known to be the first spectral graph matching method. It can solve the matching of two equal sized graphs with limited robustness. Another popular approach using pairwise constraint is spectral matching [9]. Specifically, the affinity matrix $\mathbf{A}$ is constructed based on unary and pairwise similarity, then the graph matching problem is approximated with the rank one approximation of $\mathbf{A}$, and solved with spectral method. Works that improve on spectral matching include reweighted random walk matching [23], balanced graph matching [24], probabilistic graph matching [13]. Graduated assignment [25] solves a series of one-order Taylor series approximation of the original objective function with power iteration followed by soft-assign operator. The soft-assign operator is controlled by a controlling parameter which pushes the result to a final discrete solution.

One drawback of the spectral method is that the matching problem is solved with a two-step scheme, i.e., the problem is first approximately solved in continuous domain and then projected back to discrete domain. In contrast, a path following algorithm is proposed in [26] by constructing a sequence of relaxation of the original optimization problem. Each relaxation is solved using the result of the previous as initialization and result is pushed to discrete solution in a gradual manner. Thus the constraints are employed more effectively. This path following algorithm is further improved in $[15,27,28]$. [29] also naturally imposes the discrete mapping constraint 
in the optimizing process by employing $L_{1}$ norm of the solution. An iterative approach is proposed to return an approximate discrete solution that sufficiently satisfies the constraint.

Besides pairwise constraints, other spatial relations are also used. If nonrigid deformation is present, the pairwise feature may not be preserved, especially for severe scale changes and deformation [30]. Other local geometrical constraints show better robustness such as the ones used in $[30,31,32,4]$. The binary quantized distance is used to represent the symmetric neighborhood relationship in [30]. This representation can be better preserved under scale change compared with absolute distance. Similar neighborhood preservation assumption is adopted in [31]. The outlying matches that disrupt the relationship are iteratively eliminated to derive two isomorphic neighborhood graphs in two images. [32] uses cyclic string edit distance to compute the difference of local spatial order of a correspondence, and designs a filtering strategy to rule out matches that give rise to large local spatial disorder. Local embedding is another powerful tool to describe local geometrical structure. One of the original works on local embedding is the locally linear embedding (LLE) [33] in the unsupervised learning field. The idea behind this method is to reconstruct each point with the linear combination of its $k$ neighbors. The idea is generalized to graph matching problem in [4] by constraining the linear combination to locally affine representation, which is invariant to scale, translation and affine transformation. The $l_{1}$ norm of the representation error is then used as objective function of a subgraph matching problem in an object detection application, which is solved in a linear programming framework.

\section{Graph Matching Model for Lunar Surface Correspondence}

The key points to be matched are extracted from the lunar surface images (SIFT is used in this paper). The key points in each image are modeled as a graph $\mathcal{G}=\{V, E\}$, where $V$ is the vertex set with each vertex denoting a keypoint in the image and $E$ is the edge set. Each edge carries a weight vector denoted as $w(i j)$ with $(i j) \in E$.

For a pair of lunar images, graphs are constructed as $\mathcal{G}^{1}=\left\{V^{1}, E^{1}\right\}$ and $\mathcal{G}^{2}=\left\{V^{2}, E^{2}\right\}$ with $\left|V^{1}\right|=M$ and $\left|V^{2}\right|=N$. It is assumed that $M \leq N$ without loss of generality. We denote the vertex coordinate matrices of $V^{1}$ and $V^{2}$ as $\mathbf{P}=\left[\mathbf{p}_{1}, \cdots, \mathbf{p}_{M}\right]^{T} \in \mathcal{R}^{M \times 2}$ and $\mathbf{Q}=\left[\mathbf{q}_{1}, \cdots, \mathbf{q}_{N}\right]^{T} \in \mathcal{R}^{N \times 2}$ 
respectively. The task of lunar surface correspondence is modeled as finding a $L$ sized common subgraph of $\mathcal{G}_{1}$ and $\mathcal{G}_{2}$ that preserves geometrical constraint.

In order to find the size $L$ common subgraph, the following problem needs to be solved:

$$
\begin{array}{cl}
\min & f(\mathbf{x}) \\
\text { s.t. } & \left(\mathbf{I}_{N} \otimes \mathbf{1}_{M}^{T}\right) \mathbf{x} \leq \mathbf{1}_{N} \\
& \left(\mathbf{1}_{N}^{T} \otimes \mathbf{I}_{M}\right) \mathbf{x} \leq \mathbf{1}_{M} \\
& \mathbf{1}_{M N}^{T} \mathbf{x}=L, \mathbf{x} \in\{0,1\}^{1 \times M N}
\end{array}
$$

where $\mathbf{x}$ is the row-wise replica of the $M \times N$ binary assignment matrix $\mathbf{X}$. The constraints require that $L$ nodes are selected from two graphs and match with each other. The cost function $f(\mathbf{x})$ is computed as the geometrical difference between the matched vertices. Its construction and the solution will be discussed in the following section.

\section{Graph Matching with Locally Affine-Invariance Constraints}

We first introduce the locally affine-invariance constraint to gragh matching with outliers in one graph, which is known as subgraph matching [34]. Then we generalize the locally affine-invariance constraint to graph matching problem with outliers in both graphs, which is known as common subgraph matching [35].

\subsection{Using Locally Affine-Invariance Constraint in Subgraph Matching}

In this section, a locally affine-invariance constraint and the matrix formulation of locally affine representation error are first introduced. Then the integer quadratic programming formulation of the subgraph matching problem is constructed, and solved with a path following algorithm.

\subsubsection{A Locally Affine-Invariance Constraint}

The goal of the subgraph matching problem is to find an injective correspondence function $m: V^{1} \mapsto V^{2}$ such that pairwise constraint and locally affine-invariance constraint are preserved to the most degree.

The key assumption of locally affine-invariance constraint is that the local geometry structure of a point should be preserved on its corresponding point. To characterize the local geometry structure of point $\mathbf{p}_{i}$, it is represented with the affine combination of its local neighbours such that: 


$$
\left\|\mathbf{p}_{i}-\sum_{j} W_{i j} \mathbf{p}_{j}\right\|_{F}^{2}=0
$$

where $\mathbf{W} \in \mathbb{R}^{M \times M}$ is the representation matrix with the constraints of: (1) $W_{i j}=0$ when $j \notin \mathcal{N}(i)$ and $(2) \sum_{j=1}^{M} W_{i j}=1, \mathcal{N}(i)$ denotes three non-linear nearest neighbours of $\mathbf{p}_{i} \cdot\|\cdot\|_{F}$ denotes the Frobenius norm of a matrix. $\mathbf{W}$ can be calculated by solving the following linear equations :

$$
\left[\begin{array}{ccc}
\mathbf{p}_{j_{1}} & \mathbf{p}_{j_{2}} & \mathbf{p}_{j_{3}} \\
1 & 1 & 1
\end{array}\right]\left[\begin{array}{l}
W_{i j_{1}} \\
W_{i j_{2}} \\
W_{i j_{3}}
\end{array}\right]=\left[\begin{array}{c}
\mathbf{p}_{i} \\
1
\end{array}\right]
$$

where $\mathcal{N}(i)=\left\{j_{1}, j_{2}, j_{3}\right\}$. We represent the injective correspondence function as $m: V^{1} \mapsto V^{2}$, such that $\mathbf{p}_{i}$ is matched to $m\left(\mathbf{p}_{i}\right)$. If we assume that the local transformation of the neighbour of each point $\mathbf{p}_{i}$ is subject to affine transformation, then for its corresponding point $m\left(\mathbf{p}_{i}\right)$ the representation error should be zero [4]:

$$
\left\|m\left(\mathbf{p}_{i}\right)-\sum_{j} W_{i j} m\left(\mathbf{p}_{j}\right)\right\|_{F}^{2}=0
$$

Based on this assumption, locally affine representation error on $\mathcal{G}^{2}$ is formulated as:

$$
f_{a f f}(\mathbf{X})=\|\mathbf{X Q}-\mathbf{W X Q}\|_{F}^{2}
$$

where $\mathbf{X}$ is a $M \times N$ binary assignment matrix that represents the matching function $m(\cdot)$ with $X_{i a}=1$ if $m\left(\mathbf{p}_{i}\right)=\mathbf{q}_{a}$ and $X_{i a}=0$ otherwise.

\subsubsection{Subgraph Matching Algorithm with Locally Affine-Invariance Constraint}

Besides the locally affine representation error, the energy function associated with pairwise constraint is also considered. If we denote the assignment set as $\mathcal{X}$ with $(i, a) \in \mathcal{X}$ denoting assigning $i \in V^{1}$ to $a \in V^{2}$, the pairwise energy function can be formulated as:

$$
f_{\text {pair }}(\mathbf{x})=\sum_{(i, a),(j, b) \in \mathcal{X}} d(i j, a b)
$$

Following the representation in [9] and [28], we write the pairwise cost in quadratic form:

$$
f_{\text {pair }}(\mathbf{x})=\mathbf{x}^{T} \mathbf{K}_{1} \mathbf{x}
$$


where $\mathbf{x}$ is the row-wise vectorization of $\mathbf{X}$. The pairwise dissimilarity matrix $\mathbf{K}_{1}$ is constructed as:

$$
\begin{aligned}
\mathbf{K}_{1}((i j),(a b))= & \mathbf{K}_{1}((i-1) N+a,(j-1) N+b) \\
= & \begin{cases}d(i j, a b) & \text { if } i \neq j \text { and } a \neq b, \\
k & \text { if } i=j \text { and } a=b, \\
0 & \text { otherwise }\end{cases}
\end{aligned}
$$

where $d(i j, a b)=\operatorname{distance}(w(i j), w(a b))$ measures the dissimilarity between the edges $(i j) \in E^{1}$ and $(a b) \in E^{2}$, and $k$ is a positive constant such that $\mathbf{K}_{1}$ is positive semidefinite. Here we assume that $w(i j)=w(j i)$, hence $\mathbf{K}_{1}$ is a symmetric matrix. It should be noted that different from [9] and [28], we do not take the unary matching cost into consideration.

The second term measures the locally affine representation error. We rewrite (5) with quadratic formation:

$$
\begin{aligned}
f_{a f f}(\mathbf{x}) & =\mathbf{x}^{T} \mathbf{K}_{2} \mathbf{x} \\
& =\mathbf{x}^{T}\left((I-\mathbf{W}) \otimes \mathbf{Q}^{T}\right)^{T}\left((I-\mathbf{W}) \otimes \mathbf{Q}^{T}\right) \mathbf{x}
\end{aligned}
$$

$\mathbf{K}_{2}$ is also positive semidefinite. Different from $\mathbf{K}_{1}$, the entries of $\mathbf{K}_{2}$ can be negative.

To sum up, the matching problem is formulated as:

$$
\begin{array}{cl}
\min & f(\mathbf{x})=\alpha_{1} \cdot \mathbf{x}^{T} \mathbf{K}_{1} \mathbf{x}+\alpha_{2} \cdot \mathbf{x}^{T} \mathbf{K}_{2} \mathbf{x} \\
\text { s.t. } & \left(\mathbf{I}_{N} \otimes \mathbf{1}_{M}^{T}\right) \mathbf{x}=\mathbf{1}_{N}, \\
& \left(\mathbf{1}_{N}^{T} \otimes \mathbf{I}_{M}\right) \mathbf{x} \leq \mathbf{1}_{M}, \\
& \mathbf{x} \in\{0,1\}^{M N \times 1} .
\end{array}
$$

where $\mathbf{1}$ is vector of 1 and $\mathbf{I}$ is identity matrix. Even though the $\alpha_{1} \cdot \| \mathbf{A}^{1}-$ $\mathbf{X}^{T} \mathbf{A}^{2} \mathbf{X}\left\|_{F}^{2}+\alpha_{2} \cdot\right\| \mathbf{X Q}-\mathbf{W X Q} \|_{F}^{2}$ formulation is also suitable for the subgraph matching problem, we adopt the quadratic formulation since it can avoid higher order objective function when dealing with common subgraph matching problem in Section 4.2 [15].

The problem (10) is NP-hard. Here we adopt a recently proposed path following graph matching algorithm [34] to solve it effectively.

For the objective function formulated as $\mathbf{x}^{T} \mathbf{K} \mathbf{x}$, we first derive a series of relaxed objective functions taking the form of:

$$
f_{\zeta}(\mathbf{x})=(1-\zeta) \mathbf{x}^{T} \mathbf{K} \mathbf{x}-\zeta \mathbf{x}^{T} \mathbf{x}, \quad 1 \geq \zeta \geq 0
$$


and the binary constraints $\mathbf{x} \in\{0,1\}^{M N \times 1}$ are relaxed to continuous domain $[0,1]^{M N \times 1}$. As $\zeta$ increases gradually from 0 to 1 with a step size of $d \zeta$, the objective function $f_{\zeta}(\mathbf{x})$ changes from $\mathbf{x}^{T} \mathbf{K} \mathbf{x}$ (the original convex objective function) to $-\mathbf{x}^{T} \mathbf{x}$ (a concave function). A convex concave relaxation procudure (CCRP) is realized implicitly in this process [26, 35]. For each $\zeta$ the optimization problem is solved with Frank-Wolfe algorithm [36] using the minimum of the previous iteration as starting point.

The reasons that the path following algorithm suits our application are as follows. First, it (the path following based graph matching algorithm proposed by [34]) can be directly used to solve the $L$ best assignment problem. Most existing graph matching algorithms, including the spectral based algorithms and previous path following based algorithms such as Spectral Matching[9], Balanced Graph Matching [24], and (E)PATH [26, 27], can only solve subgraph matching problem or equal-sized graph matching problem. Second, it is not an easy job for previous path following based graph matching algorithms [26, 27] to construct the convex relaxation and concave relaxation of our objective function, while [34] constructs the relaxations in a much more convenient way.

\subsubsection{Suggestions on choosing $\alpha_{1}$ and $\alpha_{2}$}

The selection of $\alpha_{1}$ and $\alpha_{2}$ can affect not only the weighting of the two kinds of cost, but also the CCRP process.

In practice, the ratio between $\mathbf{x}^{T} \mathbf{K}_{2} \mathbf{x}$ and $\mathbf{x}^{T} \mathbf{K}_{1} \mathbf{x}$ can be as large as several orders of magnitude. This makes it tedious to choose $\alpha_{1}$ and $\alpha_{2}$ through trial and error, and less intuitive to choose manually. We propose to derive $\alpha_{1}$ and $\alpha_{2}$ as:

$$
\alpha_{1}=\frac{1-\alpha}{\lambda_{1}\left(\mathbf{K}_{1}\right)}, \alpha_{2}=\frac{\alpha}{\lambda_{1}\left(\mathbf{K}_{2}\right)}
$$

where we denote $\lambda_{i}(\cdot)$ as the eigenvalues of a matrix and $\lambda_{1} \geq \ldots \geq \lambda_{M N}$, $\alpha \in[0,1]$ is the weighting parameter.

Essentially, we normalize each matrix with its principal eigenvalue. Since $\mathbf{x}^{T} \mathbf{K}_{1} \mathbf{x} \leq M \cdot \lambda_{1}\left(\mathbf{K}_{1}\right)$ and $\mathbf{x}^{T} \mathbf{K}_{2} \mathbf{x} \leq M \cdot \lambda_{1}\left(\mathbf{K}_{2}\right)$ the normalization stretches the counterparts to the same range approximately. It is computationally expensive to find the principal eigenvalue of a $(M N \times M N)$ matrix. The 1 -norm or $\infty$-norm can be used as an approximation of the upper bound of the principal eigenvalue. Even though the approximation is not precise, it 
can still serve the purpose of reducing the ratio between the counterparts significantly, and hence helps the choosing of $\alpha_{1}$ and $\alpha_{2}$.

The other benefit of this normalization is to regulate the CCRP process. The relaxed objective function becomes concave when $\zeta \geq \frac{\lambda_{1}(\mathbf{K})}{\lambda_{1}(\mathbf{K})+1}$. Generally we have $\lambda_{1}(\mathbf{K}) \gg 1$. If we use the un-normalized formulation, the

convergence of the algorithm will be hampered since $\frac{\lambda_{1}(\mathbf{K})}{\lambda_{1}(\mathbf{K})+1}$ will be too close to 1 and the objective function will become concave too slow.

\subsection{Using Locally Affine-Invariance Constraint in Common Subgraph Match- ing}

The common subgraph matching problem is a generalization of the subgraph matching problem by allowing outliers to exist in both point sets. To adapt to the common subgraph matching problem, the constraints in (10) are changed into:

$$
\left\{\begin{array}{l}
\left(\mathbf{I}_{N} \otimes \mathbf{1}_{M}^{T}\right) \mathbf{x} \leq \mathbf{1}_{N} \\
\left(\mathbf{1}_{N}^{T} \otimes \mathbf{I}_{M}\right) \mathbf{x} \leq \mathbf{1}_{M} \\
\mathbf{1}_{M N}^{T} \mathbf{x}=L, \mathbf{x} \in\{0,1\}^{M \times N} .
\end{array}\right.
$$

where $L \leq M \leq N$. The constraint explicitly requires that only $L$ best assignments should be selected. Hence it is called the " $L$ best assignment" problem [35]. When $L=M \leq N$, the problem degenerates to subgraph matching problem.

Herein, the pairwise geometrical cost can still be formulated as $\mathbf{x}^{T} \mathbf{K}_{1} \mathbf{x}$. However the quadratic formulation of locally affine representation error (9) no longer stands since it may produce unpredictable cost when some points involved in the locally affine representation are not selected in the assignments.

In this section we propose to solve the common subgraph matching problem by iteratively identifying reliable matches and solving rematching problem with locally affine representation cost built upon the relatively reliable matches.

\subsubsection{Reliable Matching Identification}

For correspondences denoted as $\left(\widetilde{V}^{1}, \widetilde{V}^{2}, m\right)$, where $\widetilde{V}^{1} \subset V^{1}$ and $\widetilde{V}^{2} \subset V^{2}$ are the point sets that have been chosen in the assignment and $m(\cdot)$ is the one-to-one assignment function, the goal of reliable matching identification is to identify subsets $V_{B}^{1} \subseteq \widetilde{V}^{1}$ and $V_{B}^{2} \subseteq \widetilde{V}^{2}$ such that the correspondences 
$\left(V_{B}^{1}, V_{B}^{2}, m_{B}\right)$ are relatively more reliable than the others. The following procedure is conducted to prune the matches:

1. Construct the representation matrix $\widetilde{\mathbf{W}}$ which represents each point $\mathbf{p}_{i} \in \widetilde{V}^{1}$ with points in $\widetilde{V}^{1}$ similar to (2):

$$
\left\|\mathbf{p}_{i}-\sum_{\mathbf{p}_{j} \in \tilde{V}^{1}} \widetilde{W}_{i j} \mathbf{p}_{j}\right\|_{F}^{2}=0
$$

2. A correspondence $\left(\mathbf{p}_{i}, m\left(\mathbf{p}_{i}\right)\right)$ and its neighbours $\left(\mathcal{N}\left(\mathbf{p}_{i}\right), m\left(\mathcal{N}\left(\mathbf{p}_{i}\right)\right)\right.$ are added to $\left(V_{B}^{1}, V_{B}^{2}, m_{B}\right)$ if they satisfy:

$$
\frac{\left\|m\left(\mathbf{p}_{i}\right)-\sum_{j \in \mathcal{N}\left(i, \widetilde{V}^{1}\right)} \widetilde{W}_{i j} m\left(\mathbf{p}_{j}\right)\right\|_{F}}{\max _{j \in \mathcal{N}\left(i, \widetilde{V}^{1}\right)}\left\|m\left(\mathbf{p}_{i}\right)-m\left(\mathbf{p}_{j}\right)\right\|_{F}} \leq \eta
$$

The idea behind this procedure is that if the locally representation error of a point is small enough, this point and its neighbours are considered to be relatively more reliable. The criterion is robust against scale change with the normalization by the longest edge connected to a point.

\subsubsection{Rematching Problem}

The objective function of the rematching problem is:

$$
f^{t}(\mathbf{x})=\alpha_{1} \cdot \mathbf{x}^{T} \mathbf{K}_{1} \mathbf{x}+\alpha_{2} \cdot \mathbf{x}^{T} \mathbf{K}_{2}^{t} \mathbf{x}
$$

with $\mathbf{K}_{2}^{t}$ updated based on the identified reliable matches. Here, we represent each point $\mathbf{p}_{i}$ in $V_{1}$ with its three non-linear nearest neighbours (except itself) $\mathbf{p}_{i^{\prime}}^{\prime}$ in $V_{B}^{1}$ as:

$$
\mathbf{p}_{i}=\sum_{\mathbf{p}_{i^{\prime}}^{\prime} \in V_{B}^{1}} W_{i i^{\prime}} \mathbf{p}_{i^{\prime}}^{\prime}
$$

where $\mathbf{W} \in R^{M \times B}$ is the representation matrix with the constraints of: (1) $W_{i i^{\prime}}=0$ when $\mathbf{p}_{i^{\prime}}^{\prime}$ is not one of nearest neighbours of $\mathbf{p}_{i}$, and (2) $\sum_{i^{\prime}=1}^{B} W_{i i^{\prime}}=$ 1. The diagonal entries of $\mathbf{K}_{2}$ are updated as:

$$
\mathbf{K}_{2}^{t}((i i),(a a))=\min \left(\left\|\mathbf{q}_{a}-\sum_{\mathbf{p}_{i^{\prime}}^{\prime} \in V_{B}^{1}} W_{i i^{\prime}} m_{B}\left(\mathbf{p}_{i^{\prime}}^{\prime}\right)\right\|_{F}^{2}, \mathbf{K}_{2}^{t-1}((i i),(a a))\right)
$$


where $\mathbf{q}_{a} \in V^{2}$ and $m_{B}\left(\mathbf{p}_{i^{\prime}}^{\prime}\right) \in V_{B}^{2}$. The current estimation of the cost of assigning $\mathbf{q}_{a}$ to $\mathbf{p}_{i}$ is the square distance between $\mathbf{q}_{a}$ and the estimated coordinate of the corresponding point of $\mathbf{p}_{i}, \sum_{\mathbf{p}_{i^{\prime}}^{\prime} \in V_{B}^{1}} W_{i i^{\prime}} m_{B}\left(\mathbf{p}_{i^{\prime}}^{\prime}\right)$. The update makes sure that the best mutual locally affine representation that ever emerged for $(i, a)$ is used to estimate the locally representation error $\mathbf{K}_{2}((i i),(a a))$. The off-diagonal entries of $\mathbf{K}_{2}$ are assigned with zero. The normalization is conducted in the first iteration of rematching, then $\alpha_{1}$ and $\alpha_{2}$ are kept for the rest iterations such that the score can be compared. The optimization algorithm used is the same as that in the subgraph matching problem.

\subsubsection{The Algorithm}

The common subgraph matching algorithm is summarized in Algorithm.1. Initially, there is no reliable matches and the matching is conducted using the pairwise cost $\mathbf{x}^{T} \mathbf{K}_{1} \mathbf{x}$ alone. Then in each iteration, the reliable matches are identified with (15) which returns the reliably assigned nodes and their correspondences. Based on this, $\mathbf{K}_{2}^{t}$ is updated and the new re-matching problem is formulated and solved. The termination of the algorithm is controlled by the score of the objective function. In our experiments, the algorithm terminates in $1 \sim 5$ iterations.

\subsubsection{Analysis of Convergence}

The proposed algorithm conducts the path following algorithm iteratively, and the path following algorithm is known to be convergent [26, 27, 28, 15]. Therefore the proposed scheme is convergent because both the update of $\mathbf{K}_{2}^{t}$ and the solving of the rematching problem monotonically decrease the objective function $f^{t}(\mathbf{x})$, and the objective function is lower-bounded by zero.

Specifically, first, by updating $\mathbf{K}_{2}^{t}$ with (18), the objective function decreases

$$
\mathbf{x}_{t}^{T} \mathbf{K}_{2}^{t} \mathbf{x}_{t} \leq \mathbf{x}_{t}^{T} \mathbf{K}_{2}^{t-1} \mathbf{x}_{t}
$$

because $\mathbf{K}_{2}^{t}((i i),(a a)) \leq \mathbf{K}_{2}^{t-1}((i i),(a a))$.

Second, in the rematching step, the new matching problem with the updated $\mathbf{K}_{2}^{t}$ is solved. Ideally, if the global solution $\mathbf{x}^{*}$ of the rematching problem is found, it is straightforward that $f^{t}\left(\mathbf{x}^{*}\right) \leq f^{t}\left(\mathbf{x}^{t-1}\right)$. However, since the common subgraph matching problem is NP-hard and the adopted algorithm [34] is an inexact graph matching algorithm, the solution $\mathbf{x}_{t}$ may not 
$\overline{\text { Algorithm } 1 \text { Common subgraph matching with locally affine-invariance }}$ constraint

Require: Two graphs $G^{1}$ and $G^{2}$;

Ensure: The assignment vector $\mathbf{x}$;

1: Initialize: $V_{B}^{1}=\emptyset, V_{B}^{2}=\emptyset, t=0$

2: Construct $\mathbf{K}_{1}$ with (8)

3: Solve the initial matching with the objective function $f^{0}(\mathbf{x})=\mathbf{x}^{T} \mathbf{K}_{1} \mathbf{x}$

4: repeat

5: $\quad t \leftarrow t+1$

6: $\quad\left(V_{B}^{1}, V_{B}^{2}\right)=$ FindReliableMatches $\left(V^{1}, V^{2}, \mathbf{x}_{t}\right)$, based on the criterion of (15)

7: $\quad$ Update $\mathbf{K}_{2}^{t}$ with (18), and $f^{t}(\mathbf{x})=\alpha_{1} \cdot \mathbf{x}^{T} \mathbf{K}_{1} \mathbf{x}+\alpha_{2} \cdot \mathbf{x}^{T} \mathbf{K}_{2}^{t} \mathbf{x}$

8: $\quad$ Solve the rematching problem and derive the result $\mathbf{x}_{t}$

9: $\quad$ if $f^{t}\left(\mathbf{x}_{t}\right)>f^{t}\left(\mathbf{x}_{t-1}\right)$ then

10: $\quad$ return $\mathrm{x}_{t-1}$

11: $\quad$ end if

12: until $\left|f^{t}\left(\mathbf{x}_{t}\right)-f^{t}\left(\mathbf{x}_{t-1}\right)\right|<\epsilon$

13: return $\mathrm{x}_{t}$

be the global optimal solution and possibly causes $f^{t}\left(\mathbf{x}_{t}\right)>f^{t}\left(\mathbf{x}_{t-1}\right)$. To make sure that the algorithm converges, an artificial criterion is added: if $f^{t}\left(\mathbf{x}_{t}\right)>f^{t}\left(\mathbf{x}_{t-1}\right)$, then the algorithm terminates, and $\mathbf{x}_{t-1}$ is returned as the final result.

\subsubsection{Analysis of Computational Complexity}

For subgraph matching in Section 4.1, the computation of the locally affine representation involves a complexity of $O\left(M^{2}\right)$ because it needs to find the nearest neighbour of each vertex. The optimization by the path following algorithm involves a complexity of $O\left(M^{2} N\right)$. Hence the overall complexity is $O\left(M^{2} N\right)$.

For each iteration of common subgraph matching in section 4.2, updating $\mathbf{K}_{2}$ involves a complexity of $O(M N L)$, smaller than the complexity of its optimization, which is also $O\left(M^{2} N\right)$ if a quick solution in [15] is adopted. Hence the overall complexity is still $O\left(M^{2} N\right)$. Please refer to the supplementary material for experimental results regarding running time. 


\section{Experiments}

In this section, we report experimental results on several datasets to compare our algorithm to state-of-the-art methods. The proposed subgraph matching algorithm is denoted as AF and the common subgraph matching algorithm is denoted as AFL. These two algorithms are compared with state-of-the-art methods including: SM [9], GA [25], PGM [6], and RRWM [23].

The algorithms are first tested on synthetic graphs with various setups of outlier and noise. Then experiments are conducted on several simulated and real-world images datasets, including a lunar surface dataset. Furthermore, the results are analyzed with paired z-test and Friedman test. Paired z-test is used to compare the mean accuracy of considered methods under specific outlier and noise. The null hypothesis is that two methods perform equally and rejection of the null hypothesis means that the pair of methods perform significantly different. A Friedman test [37] is a nonparametric statistical test for multiple methods and multiple test groups. The null hypothesis for this test is that all the methods perform equally, and rejection of the null hypothesis means that there is at least one pair of methods with significantly different performance. When the null hypothesis of Friedman test is rejected, we can proceed with a post-hoc Nemenyi test [37] to find out which method has the best performance.

We use same undirected edge feature in all the experiments except the third real world image set in which the pairwise edge distances have been given. In particular, the normalized pairwise distance and direction between the connected nodes are used as edge features $w_{i j}$. To be specific, for nodes $\mathbf{p}_{i}, \mathbf{p}_{j}$, the offset vector of the edge is denoted as $\mathbf{r}_{i j}=\mathbf{p}_{j}-\mathbf{p}_{i}$. The normalized pairwise distance is calculated as $w_{d i s}(i j)=\left|\mathbf{r}_{i j}\right| / \max _{i, j}\left(\left|\mathbf{r}_{i j}\right|\right)$. The direction of the edge is the acute angle between the offset vector and $e_{x}$ [11] defined by:

$$
w_{d i r}(i j)=\frac{1}{\pi} \arcsin \frac{\mathbf{r}_{i j} \times e_{x}}{\left|\mathbf{r}_{i j}\right|}
$$

$e_{x}$ is the unit vector along x-axis. The edge feature is then constructed as $w_{i j}=\left[w_{d i s}(i j), w_{\text {ori }}(i j)\right]$. The pairwise dissimilarity $d(i j, a b)$ is computed by $d(i j, a b)=\left\|w_{i j}^{1}-w_{a b}^{2}\right\|_{2}^{2}$. In GA, SM, PGM and RRWM, the pairwise similarity is computed as $\exp \left(-\frac{d(i j, a b)}{\delta}\right)$. GA and SM are designed for the subgraph 
matching problem. To adapt them to the common subgraph matching problem, their continuous solution $\mathbf{x}_{C} \in \mathcal{C}$ is back projected to the discrete domain $\mathcal{D}$ by the linear programming technique:

$$
\begin{aligned}
& \mathbf{x}_{D}=\arg \max \mathbf{x}_{C}^{T} \mathbf{x}_{D} \\
& \text { s.t. } \quad \mathbf{x}_{D} \in \mathcal{D}
\end{aligned}
$$

where $\mathcal{D}$ is generated by (13).

All the algorithms are implemented with Matlab R2014a. The balancing parameter $\alpha$ is set to be 0.5 in both subgraph matching problem and common subgraph matching problem, the kernel width $\delta$ is set to be 0.15 in our experiments, and the threshold $\eta$ used in reliable matching identification is set to be 0.1 , the step size $d \zeta$ is set to be 0.01 .

In the experiments, the matching accuracy is compared, which is defined as follows:

1. Accuracy in the subgraph matching problem is calculated as the ratio of the number of correct matches to the size of the smaller graph $M$ :

$$
\text { accuracy }=\frac{\# \text { CorrectMatches }}{M}
$$

2. Accuracy in the common subgraph matching problem is calculated as the ratio of the number of correct matches to the predefined size of common subgraph $L$ :

$$
\text { accuracy }=\frac{\# \text { CorrectMatches }}{L}
$$

\subsection{Synthetic Graphs Matching}

The first experiment is based on synthetic graphs to validate the effectiveness of the proposed algorithms, similar to previous works [6, 25]. Firstly, $L=15$ points are uniformly sampled from 2 -D square of side length 1 as inliers, then $N_{\text {out } 1}$ outliers uniformly sampled from the same square are added to them to construct graph $\mathcal{G}_{1}, N_{\text {out2 }}$ outliers and noise following $N(0, \sigma)$ are added to them to construct graph $\mathcal{G}_{2}$. Six protocols are used to test the algorithms:

1. $N_{\text {out } 1}=0, N_{\text {out } 2}=5, \sigma$ increases from 0 to 0.1 with a step size 0.01 (Fig. 1(a)). 
2. $N_{\text {out } 1}=N_{\text {out } 2}=5, \sigma$ increases from 0 to 0.1 with a step size 0.01 (Fig. $1(\mathrm{~b}))$.

3. $\sigma=0.02, N_{\text {out } 1}=0, N_{\text {out } 2}$ increases from 0 to 10 with a step size 1 (Fig. 1(c)).

4. $\sigma=0.02, N_{\text {out } 1}=N_{\text {out } 2}$ increases from 0 to 10 with a step size 1 (Fig. $1(\mathrm{~d}))$.

5. $\sigma=0.05, N_{\text {out } 1}=0, N_{\text {out } 2}$ increases from 0 to 10 with a step size 1 (Fig. 1(e)).

6. $\sigma=0.05, N_{\text {out } 1}=N_{\text {out } 2}$ increases from 0 to 10 with a step size 1 (Fig. $1(\mathrm{f}))$.

According to each of the protocols, 50 pairs of graphs are randomly generated. The point correspondence results are shown in Fig. 1. Half of the experiments are for subgraph matching problem (the left column of Fig. 1), and half are for common subgraph matching problem (the right column of Fig. 1). Based on the results, we can observe that in most occasions AF and AFL show similar performance on subgraph matching problems, which means the iterative procedure of AFL can reliably estimate the locally affine representation error. AF outperforms other algorithms on subgraph matching problems. In some cases (such as when \#out2=10 in Fig. 1(e)), AFL is even better than AF. This is probably because that the formulation of AF involves a more complicated matrix $\mathbf{K}_{2}$ compared with the diagonal one in AFL, which makes it harder to find its optimal solution. In the common subgraph matching problems, AFL also outperforms other algorithms.

The significance of the difference between the proposed algorithms and the other algorithms is also evaluated on several setups by z-score and pvalue as shown in Table 1-4. Virtually all the algorithms perform similarly on these groups of tests since there is no single algorithm that significantly outperforms all others. The only statistically significantly different pair is hypothesis 1 (AFL vs. SM) in Table 2-4 where the p-value is smaller than 0.05 . 


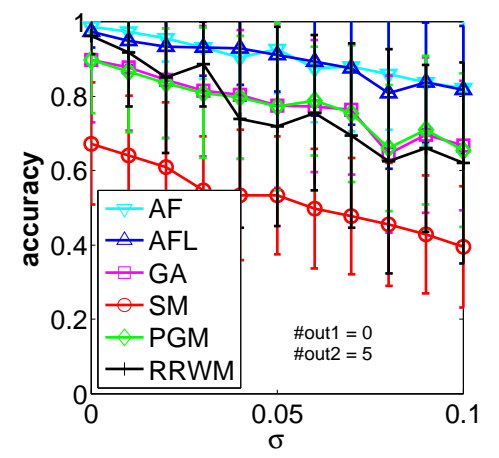

(a)

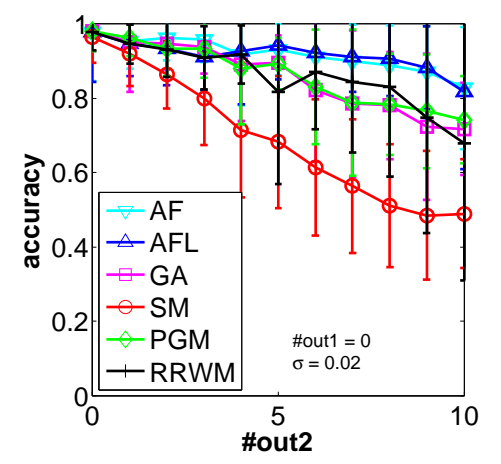

(c)

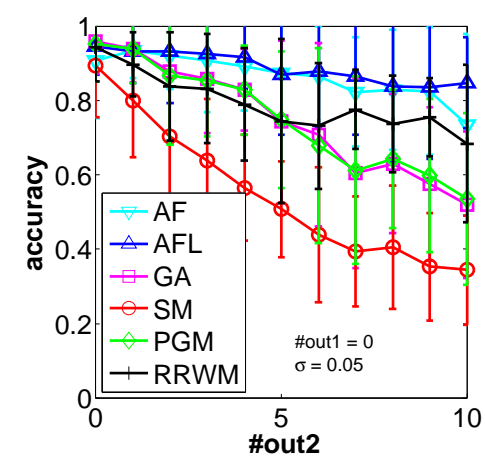

(e)

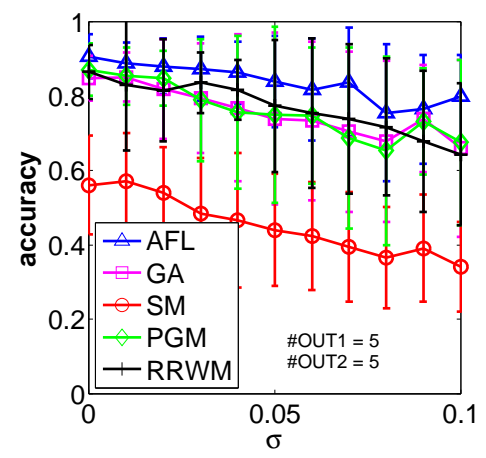

(b)

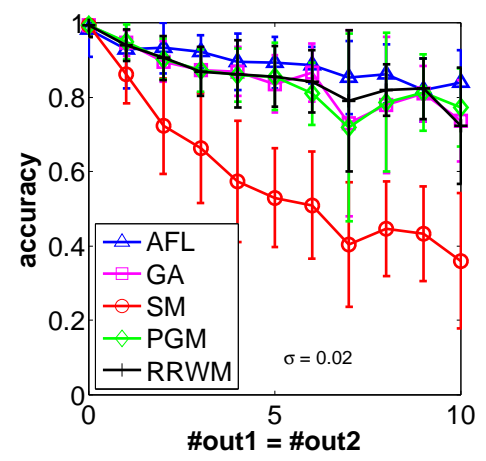

(d)

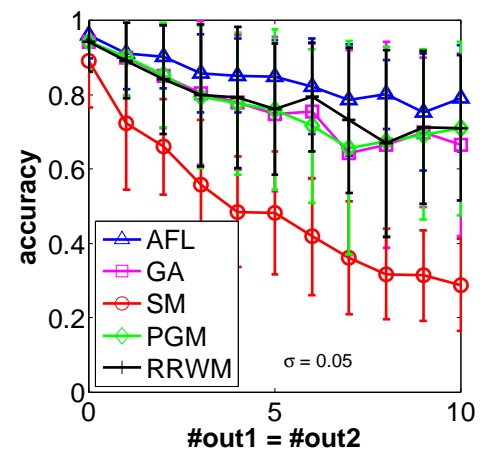

(f)

Figure 1: Matching accuracy on synthetic graphs generated by six protocols, summarized from 50 random runs on each protocol. 
Table 1: Significant pairwise difference test when $N_{\text {out } 1}=0, N_{\text {out } 2}=5$ and $\sigma=$ 0.1 .

\begin{tabular}{cccc}
\hline i & hypothesis & z-score & p-value \\
\hline 1 & AFL vs. SM & 1.783 & 0.0746 \\
2 & AF vs. SM & 1.669 & 0.0951 \\
3 & GA vs. SM & 1.143 & 0.2530 \\
4 & PGM vs. SM & 0.988 & 0.3232 \\
5 & RRWM vs. SM & 0.714 & 0.4752 \\
6 & AFL vs. RRWM & 0.615 & 0.5386 \\
7 & AFL vs. GA & 0.607 & 0.5439 \\
8 & AFL vs. PGM & 0.604 & 0.5458 \\
9 & AF vs. RRWM & 0.601 & 0.5478 \\
10 & AF vs. PGM & 0.583 & 0.5599 \\
11 & AF vs. GA & 0.581 & 0.5612 \\
12 & GA vs. RRWM & 0.149 & 0.8816 \\
13 & PGM vs. RRWM & 0.102 & 0.9188 \\
14 & GA vs. PGM & 0.049 & 0.9609 \\
15 & AF vs. AFL & 0.015 & 0.9880 \\
\hline
\end{tabular}

Table 3: Significant pairwise difference test when $N_{\text {out } 1}=5, N_{\text {out } 2}=5$ and $\sigma=$ 0.1 .

\begin{tabular}{cccc}
\hline $\mathrm{i}$ & hypothesis & z-score & p-value \\
\hline 1 & AFL vs. SM & 2.762 & 0.0057 \\
2 & RRWM vs. SM & 1.335 & 0.1819 \\
3 & PGM vs. SM & 1.319 & 0.1872 \\
4 & GA vs. SM & 1.189 & 0.2344 \\
5 & AFL vs. RRWM & 0.697 & 0.4858 \\
6 & AFL vs. GA & 0.526 & 0.5989 \\
7 & AFL vs. PGM & 0.492 & 0.6227 \\
8 & PGM vs. RRWM & 0.109 & 0.9132 \\
9 & GA vs. RRWM & 0.052 & 0.9585 \\
10 & PGM vs. GA & 0.049 & 0.9609 \\
\hline
\end{tabular}

Table 2: Significant pairwise difference test when $N_{\text {out } 1}=0, N_{\text {out } 2}=10$ and $\sigma=0.05$.

\begin{tabular}{cccc}
\hline i & hypothesis & z-score & p-value \\
\hline 1 & AFL vs. SM & 2.639 & 0.0083 \\
2 & AFL vs. GA & 1.415 & 0.1571 \\
3 & AF vs. SM & 1.376 & 0.1688 \\
4 & RRWM vs. SM & 1.317 & 0.1878 \\
5 & AFL vs. PGM & 1.197 & 0.2313 \\
6 & GA vs. SM & 0.714 & 0.4752 \\
7 & PGM vs. SM & 0.7 & 0.4839 \\
8 & AF vs. GA & 0.688 & 0.4915 \\
9 & AFL vs. RRWM & 0.666 & 0.5054 \\
10 & AF vs. PGM & 0.595 & 0.5518 \\
11 & RRWM vs. GA & 0.568 & 0.5700 \\
12 & RRWM vs. PGM & 0.474 & 0.6355 \\
13 & AFL vs. AF & 0.415 & 0.6781 \\
14 & AF vs. RRWM & 0.156 & 0.8760 \\
15 & PGM vs. GA & 0.053 & 0.9577 \\
\hline
\end{tabular}

Table 4: Significant pairwise difference test when $N_{\text {out } 1}=10, N_{\text {out } 2}=10$ and $\sigma=0.05$.

\begin{tabular}{cccc}
\hline i & hypothesis & z-score & p-value \\
\hline 1 & AFL vs. SM & 2.663 & 0.0077 \\
2 & RRWM vs. SM & 1.827 & 0.0677 \\
3 & PGM vs. SM & 1.596 & 0.1105 \\
4 & GA vs. SM & 1.37 & 0.1707 \\
5 & AFL vs. GA & 0.441 & 0.6592 \\
6 & AFL vs. RRWM & 0.331 & 0.7406 \\
7 & AFL vs. PGM & 0.298 & 0.7657 \\
8 & RRWM vs. GA & 0.144 & 0.8855 \\
9 & PGM vs. GA & 0.13 & 0.8966 \\
10 & RRWM vs. PGM & 0.004 & 0.9968 \\
\hline
\end{tabular}

To rank the methods globally, Friedman test is conducted on the subgraph matching and common subgraph matching problem respectively. The test is conducted based on the average accuracy for each setting of noise and outlier. The Friedman test proves that the null hypothesis can be rejected for both problems, $\chi^{2}=67.3, p$-value $<0.05$ for subgraph matching, $\chi^{2}=$ $72.1, p$-value $=<0.05$ for common subgraph matching. The mean rank of each method is also derived. We proceed with the Nemenyi post-hoc test to determine which method has the best performance. The performance of two methods is significantly different if the corresponding mean ranks differ by 
at least the critical difference:

$$
C D=q_{0.05} \sqrt{\frac{k(k+1)}{6 n}}
$$

where the critical value $q_{0.05}$ can be found in [37], $k$ is the number of methods to compare, and $n$ is the number of groups. Each group represents tests with a specific setting of outlier and noise. We then determine if one algorithm is better than another by comparing the difference between their mean ranks with the critical difference $C D$, as shown in Table 5 and Table 6 . The winequal-loss 4-1-0 for AF in Table 5 means that the algorithm is better than 4 methods, equal to 1 method, and worse than 0 methods.

For the subgraph matching, the critical difference is $C D=1.33$. AF ranks the first followed by AFL ranking the second. The difference between them is not significant. GA, PGM and RRWM give similar performance with no significant difference among them. SM ranks the last with the worst performance. For the common subgraph matching problem, the critical difference is $C D=1.08$ and AFL ranks first among all considered methods.

Table 5: Friedman test and post-hoc Nemenyi test result for subgraph matching problem on synthetic graphs.

\begin{tabular}{ccccccc}
\hline Method & AF & AFL & GA & PGM & RRWM & SM \\
\hline Mean rank & 1.87 & 1.94 & 3.50 & 3.68 & 4.05 & 5.86 \\
\hline win-equal-loss & $4-1-0$ & $4-1-0$ & $1-2-2$ & $1-2-2$ & $1-2-2$ & $0-0-5$ \\
\hline
\end{tabular}

Table 6: Friedman test and post-hoc Nemenyi test result for common subgraph matching problem on synthetic graphs.

\begin{tabular}{cccccc}
\hline Method & AFL & RRWM & PGM & GA & SM \\
\hline Mean rank & 1 & 2.73 & 3.05 & 3.23 & 5 \\
\hline win-equal-loss & $4-0-0$ & $1-2-1$ & $1-2-1$ & $1-2-1$ & $0-0-4$ \\
\hline
\end{tabular}

\subsection{CMU House Sequence}

The CMU house image dataset consists of 111 frames of a house. Each of them has been manually labeled with 30 landmarks. 


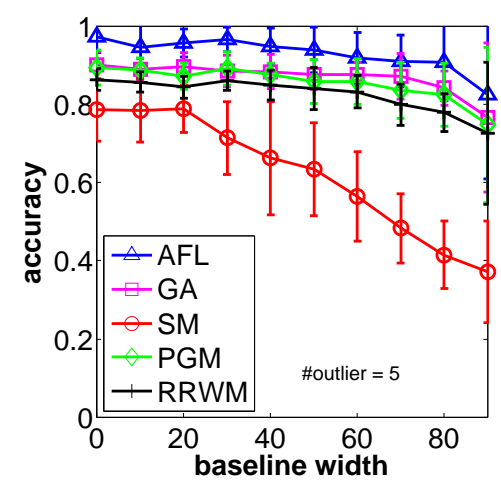

(a)

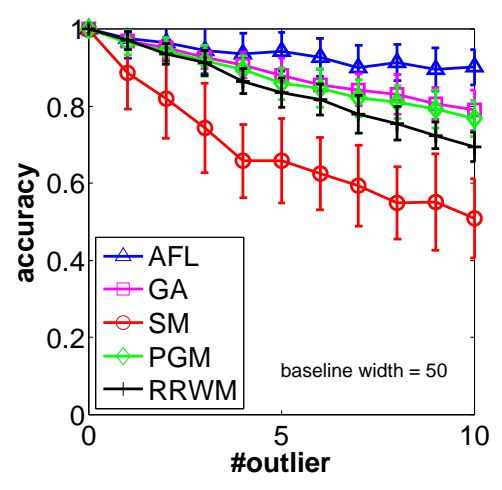

(b)

Figure 2: Comparison of common subgraph matching algorithms on the house sequence. Comparison of several algorithms with respect to (a) baseline width and (b) \#outlier.

Table 7: Significant pairwise difference test on CMU house sequence, baseline width $=90, \#$ outlier $=5$

\begin{tabular}{cccc}
\hline i & hypothesis & z-score & p-value \\
\hline 1 & AFL vs. SM & 1.801 & 0.0717 \\
2 & GA vs. SM & 1.714 & 0.0865 \\
3 & PGM vs. SM & 1.582 & 0.1136 \\
4 & RRWM vs. SM & 1.58 & 0.1141 \\
5 & AFL vs. RRWM & 0.353 & 0.7241 \\
6 & AFL vs. PGM & 0.267 & 0.7895 \\
7 & AFL vs. GA & 0.202 & 0.8399 \\
8 & GA vs. RRWM & 0.158 & 0.8745 \\
9 & PGM vs. RRWM & 0.08 & 0.9362 \\
10 & GA vs. PGM & 0.073 & 0.9418 \\
\hline
\end{tabular}

Table 8: Significant pairwise difference test on CMU house sequence, baseline width $=50, \#$ outlier $=10$

\begin{tabular}{cccc}
\hline $\mathrm{i}$ & hypothesis & z-score & p-value \\
\hline 1 & AFL vs. RRWM & 3.494 & $4.7584 \mathrm{e}-04$ \\
2 & AFL vs. SM & 3.478 & $5.0517 \mathrm{e}-04$ \\
3 & GA vs. SM & 2.427 & 0.0152 \\
4 & PGM vs. SM & 2.286 & 0.0223 \\
5 & AFL vs. PGM & 2.038 & 0.0415 \\
6 & RRWM vs. SM & 1.693 & 0.0905 \\
7 & AFL vs. GA & 1.627 & 0.1037 \\
8 & GA vs. RRWM & 1.47 & 0.1416 \\
9 & PGM vs. RRWM & 1.213 & 0.2251 \\
10 & GA vs. PGM & 0.301 & 0.7634 \\
\hline
\end{tabular}

Table 9: Friedman test and post-hoc Nemenyi test result for the house sequence.

\begin{tabular}{cccccc}
\hline Method & AFL & GA & PGM & RRWM & SM \\
\hline Mean rank & 1.16 & 2.16 & 2.98 & 3.80 & 4.91 \\
\hline win-equal-loss & $3-1-0$ & $2-2-0$ & $1-2-1$ & $0-2-2$ & $0-0-4$ \\
\hline
\end{tabular}

Same number of randomly chosen outliers are added to the ground truth landmarks of both images to generate $G^{1}$ and $G^{2}$. Similarly, we compare AFL against four state-of-the-art methods.

We first compare the accuracies as the baseline width increases from 0 to 90 with the number of outliers set to be 5 . Then the accuracies are compared with respect to the number of outliers from 0 to 10 when the baseline width 


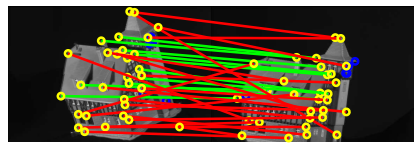

(a) SM

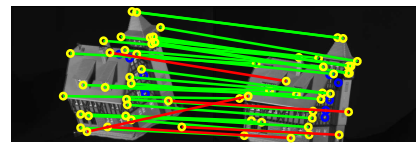

(b) GA

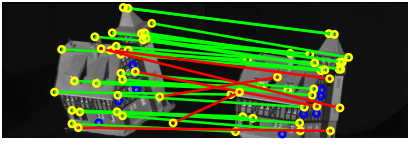

(c) RRWM

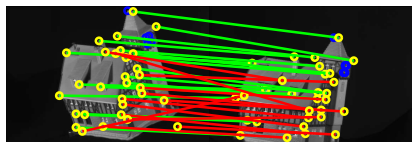

(d) PGM

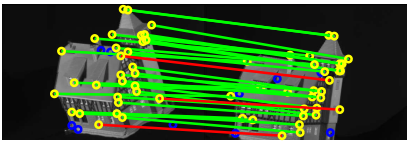

(e) AFL

Figure 3: Matching samples on CMU house sequence.

is set to be 50. For each setup, the experiment is repeated on 50 pairs of images. The results of matching accuracy with respect to baseline width and number of outliers are shown in Fig. 2. In all setups, the average accuracy of AFL is the highest. In addition, it is interesting to notice that AFL performs particularly well with large number of outliers. It is because that many key points on the house images fall on a plane in 3D space, which makes their transformation affine invariant. AFL can benefit from this property for rejecting the outliers.

The statistical comparison results with baseline width 90, \#outlier 5, and with baseline width 50, \#outlier 10 are shown in Table 7-8. All the algorithms perform similarly on these groups of test. The only exception is AFL in Table 8 which significantly outperforms PGM, RRWM, and SM when considering baseline width $=50$ and \# outlier $=10$.

The Friedman test is also conducted over different settings of outlier and noise. The results indicate that the null hypothesis can be rejected, $\chi^{2}=$ 79.1, p-value $<0.05$. In the post-hoc Nemenyi test the critical distance is $C D=1.33$. The mean ranks and result of comparisons are shown in Table 9. We can observe that AFL ranks first with mean rank 1.16. GA is also competitive for this dataset and gives similar performance to AFL. The difference between GA, PGM, and RRWM is still insignificant.

Some matching results with baseline width 90 and \#outliers 5 are shown in Fig. 3.

\subsection{Real Images Matching}

In the first experiment on real image, we use the car and motorbike image dataset to test both AF and AFL on subgraph matching problem. The car 
and motorbike image dataset was created in [38]. The dataset consists of 30 pairs of car images and 20 pairs of motorbike images along with ground truth labels. The graphs are built in the following way. For each pair of images, the smaller graph $G^{1}$ is built on the ground truth landmarks of the first image. Randomly chosen outliers are added to the ground truth landmarks of the second image to build the larger graph $G^{2}$. The matching accuracy is compared with the number of outliers changing from 0 to 10 with step size of 1 . The result of matching accuracy is shown in Fig. 4.

For both car and motorbike images AF and AFL still consistently outperform other methods even though the keypoints in this dataset suffer from more deformation than that of the house sequence, as shown in Fig. 4. RRWM, GA and PGM performs similarly when the number of outliers is small. When the number of outliers becomes bigger RRWM performs worse. It can also be observed from Fig. 4 that the standard deviations for all methods are very high. Some matching samples with AF are shown in Fig. 5 .

The Friedman test is also conducted over different number of outliers for both datasets. The result indicates that there is significant difference in considered methods for both datasets, $\chi^{2}=32.6, p$-value $<0.05$ for the car dataset, and $\chi^{2}=50.3, p$-value $<0.05$ for the motorbike dataset. The critical difference for Nemenyi test is $C D=2.27$ for both datasets. The results from Friedman test and Nemenyi test are shown in Table 10 and Table 11. In both datasets AFL ranks first and AF ranks second. It can also be observed that with higher level of deformation in this dataset, the difference between the proposed methods and others become smaller. For instance, AF performs equally with two other methods except for AFL in the motorbike dataset. This suggests that higher level deformation may weaken the effectiveness of locally affine-invariance constraints, but the matching algorithm can still benefit from the constraints. 


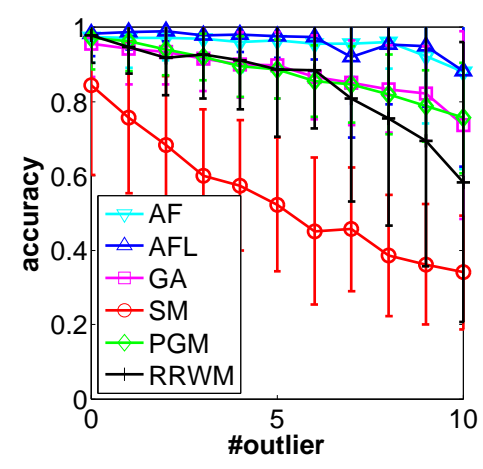

(a) car

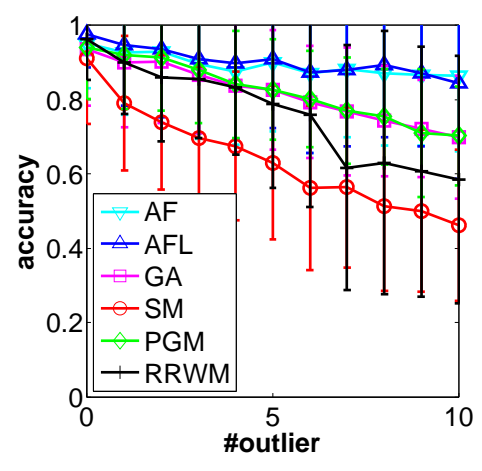

(b) motorbike

Figure 4: Comparison of subgraph matching algorithms on the (a) car and (b) motorbike dataset.
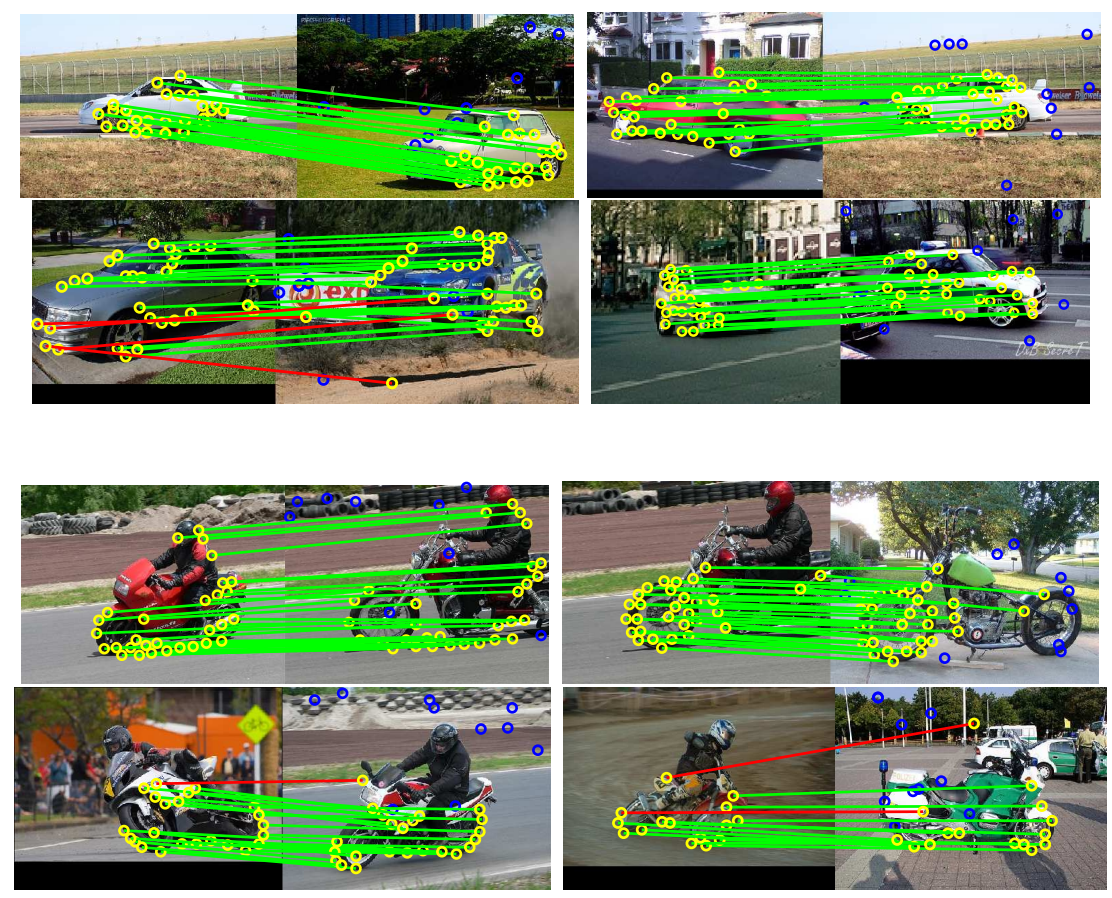

Figure 5: Matching samples on car dataset (upper two rows) and motorbike dataset (lower two rows) with $\mathrm{AF}$. 
Table 10: Friedman test and post-hoc Nemenyi test result for the car dataset.

\begin{tabular}{ccccccc}
\hline Method & AFL & AF & RRWM & GA & PGM & SM \\
\hline Mean rank & 1.18 & 1.90 & 3.91 & 3.91 & 4.09 & 6 \\
\hline win-equal-loss & $4-1-0$ & $1-4-0$ & $0-4-1$ & $0-4-1$ & $0-4-1$ & $0-3-2$ \\
\hline
\end{tabular}

Table 11: Friedman test and post-hoc Nemenyi test result for the motorbike dataset.

\begin{tabular}{ccccccc}
\hline Method & AFL & AF & PGM & GA & RRWM & SM \\
\hline Mean rank & 1.18 & 1.91 & 3.20 & 4 & 4.73 & 6 \\
\hline win-equal-loss & $3-2-0$ & $2-3-0$ & $1-4-0$ & $0-4-1$ & $0-3-2$ & $0-0-5$ \\
\hline
\end{tabular}

The second experiment is conducted on the Zurich Building Image Database (ZuBud) [39] to test the performance of AFL, SM, PGM, and RRWM on the common subgraph matching problem. The dataset contains images of different viewpoints acquired from various scenes. 30 pair of images are selected for this experiment, including the images shown in Fig. 6. Feature points are detected on each image pair. As we can observe, the images in each pair contain many repetitive patterns which means that there are multiple possible correspondences for each keypoint. To examine the algorithms on the datasets, we first manually labeled 30 ground truth correspondences for each pair of images (since there lacks ground truth label for this dataset). Then we extend each point set to 60 points by adding an additional nearest neibghbor in SIFT feature space for each keypoint (other than the points that have already existed in the point set) as outliers. We allow $K=\{5,10,15,20\}$ initial candidate correspondences for each keypoint. With larger $K$, the matching task becomes harder. Some matching results are shown in Fig. 7. The average matching accuracies are shown in Table 12. AFL works better than PGM and gives the best performance in all conditions. When $K=5$, SM performs better than GA, PGM and RRWM. While with larger $K$, the performance of SM becomes worse and PGM generally performs better than other algorithms.

Table 12: Matching Results on Zubud Dataset

\begin{tabular}{cccccc}
\hline & AFL & GA & SM & PGM & RRWM \\
\hline $\mathrm{K}=5$ & 0.8217 & 0.610 & 0.7083 & 0.6867 & 0.5917 \\
\hline $\mathrm{K}=10$ & 0.8217 & 0.6217 & 0.5783 & 0.6733 & 0.5783 \\
\hline $\mathrm{K}=15$ & 0.8017 & 0.5683 & 0.5050 & 0.6500 & 0.6133 \\
\hline $\mathrm{K}=20$ & 0.7200 & 0.5067 & 0.4967 & 0.6033 & 0.5683 \\
\hline
\end{tabular}

The third experiment is conducted on the challenging real world image set created in [23]. The dataset also contains keypoints, pairs-wise edge distance, 

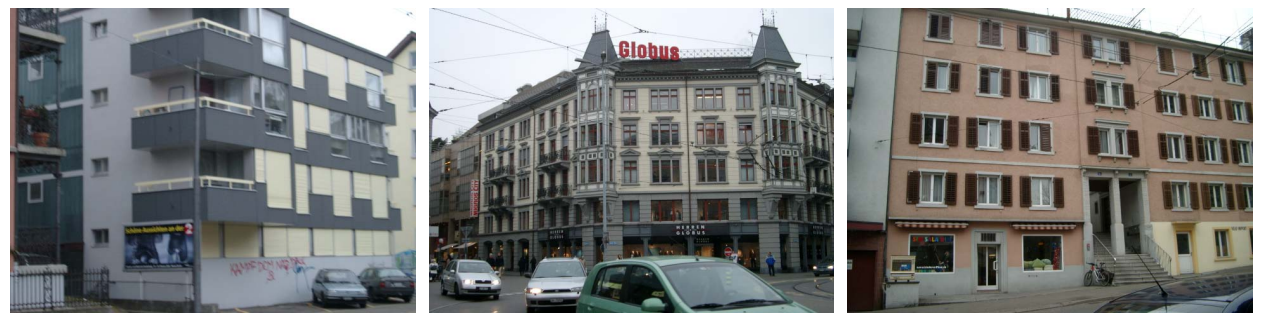

Figure 6: Image samples in Zubud dataset [39].
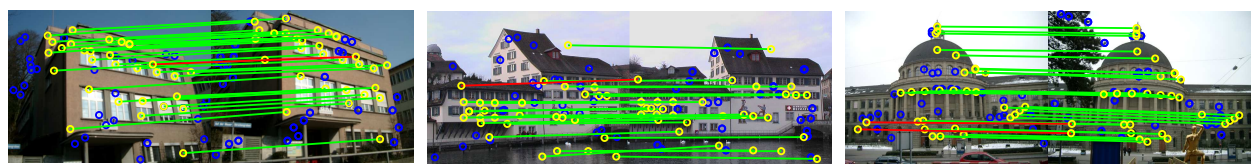

Figure 7: Matching samples on Zubud dataset [39] with AFL.

and ground truth labels. Specifically, the keypoints are detected with MSER detector [17]. The 128-dim SIFT descriptor [5] is used to derive the candidate correspondences. Specifically, multiple correspondences are allowed for each keypoint by collecting the feature pairs that have closer distance in SIFT feature space than a threshold of 0.6. The pairwise dissimilarity $d(i j, a b)$ is calculated using the mutual projection error function used in [40]. The ground truths labeled labeled for each pair.

The goal of this task is to find the common subgraph between two graphs. The size of the common subgraph is set to be the size of the ground truth pairs, and the accuracy is computed and compared using AFL, SM, GA, RRWM, and PGM. The average matching accuracy of the proposed algorithm is higher than others as shown in Table 13. Some representative matching examples are shown in Fig. 8. It should be noted that matching the image pairs is challenging because the number of outliers is larger than the number of inliers in most cases. 


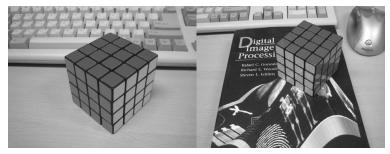

Image pair

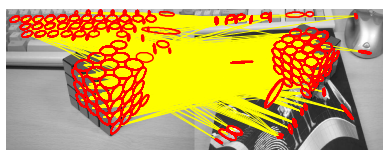

Candidate matches: 780

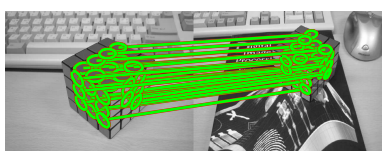

$\operatorname{AFL}(24 / 24)$

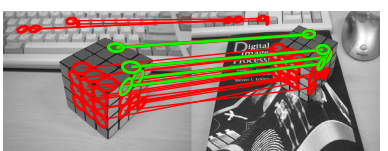

$\mathrm{GA}(4 / 24)$

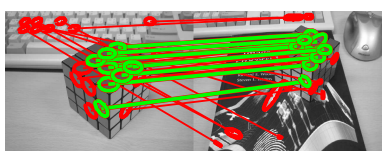

$\operatorname{PGM}(9 / 24)$

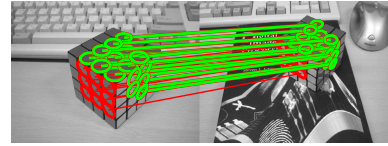

$\operatorname{SM}(15 / 24)$

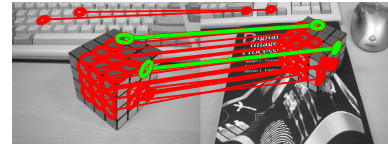

$\operatorname{RRWM}(2 / 24)$

(a)

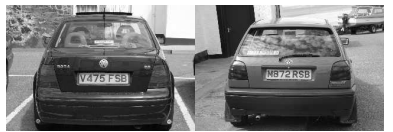

Image pair

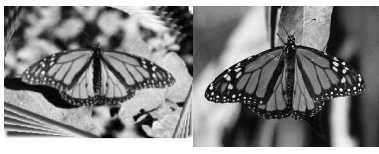

Image pair

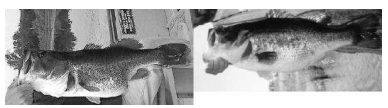

Image pair

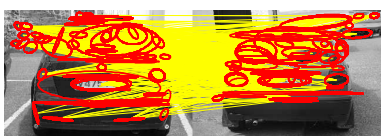

Candidate matches: 814

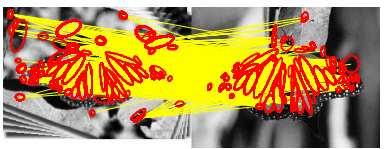

Candidate matches: 838

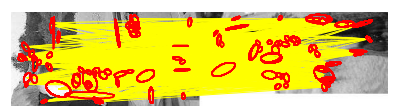

Candidate matches: 798

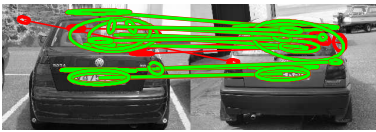

$\operatorname{AFL}(11 / 26)$

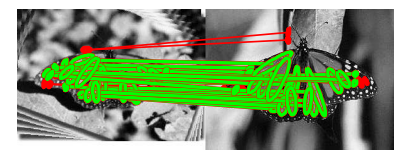

$\operatorname{AFL}(22 / 31)$

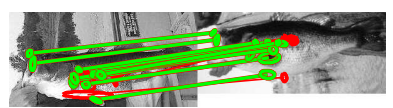

$\operatorname{AFL}(12 / 18)$

(b)

Figure 8: Some results of common subgraph matching on the real word images [23]. Yellow lines indicate the initial candidate matches. Green lines indicate the correct matches while red lines indicate wrong matches. The number of correctly matched pairs is also shown as (\#correct matched/size of common subgraph).

Table 13: Average matching accuracy on the real image set [23].

\begin{tabular}{cccccc}
\hline Method & AFL & RRWM & PGM & GA & SM \\
\hline Avg. of accuracy & 0.459 & 0.407 & 0.248 & 0.380 & 0.390 \\
\hline
\end{tabular}


The last experiment tests the performance of the common subgraph matching algorithm on the lunar surface images. The lunar surface image dataset consists of 30 pairs of images acquired by Apolo 16 and Chinese Yutu rover. In each pair of images, 30 correspondences are labeled as ground truth. The accuracies are compared as the number of the outliers increases from 0 to 20 by a step 2 . As observed in Fig. 9, AFL consistently outperforms other algorithms. AFL can match more than $80 \%$ of the points when there are 20 outliers, while the performance of other methods drastically drops when the number of outliers increases.

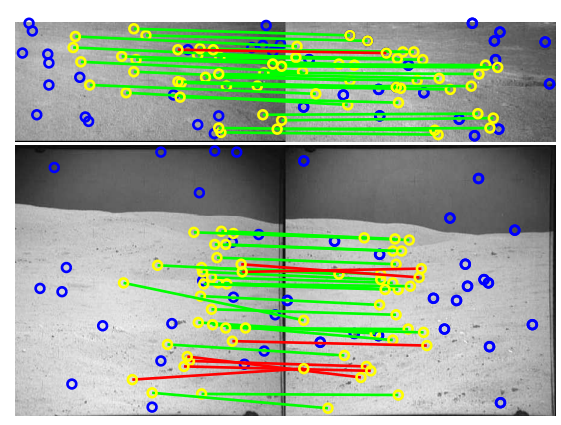

(a)

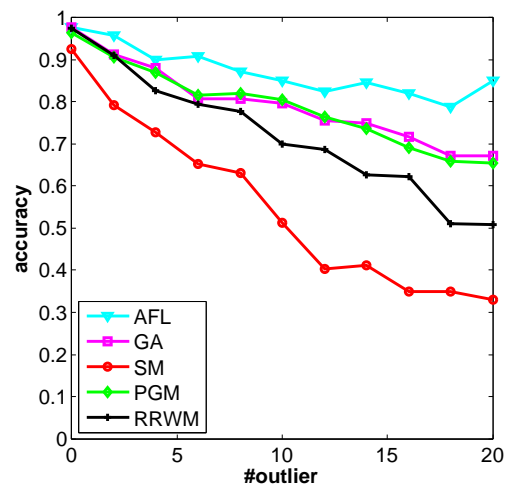

(b)

Figure 9: Comparison of common subgraph matching algorithms on the lunar surface images dataset. (a) Example pairs of lunar images with the correspondences generated by AFL when \#outlier=20, green lines indicate correct matches. (b) Performance as a function of \#outlier.

\section{Conclusion}

In this paper, we introduced a keypoint correspondence algorithm for lunar images based on graph matching. The locally affine-invariance constraint is utilized to tackle point ambiguity caused by repetitive patterns and outliers. Besides subgraph matching algorithm, we also solved the common subgraph matching problem based on reliable matches identification and rematching. The experiments demonstrated that it outperforms the stateof-the-art methods not only on the lunar surface image dataset, but also on several benchmark datasets. 


\section{Acknowledgement}

This work is supported by the National Science Foundation of China (NSFC) (grants 61375005, 61033011, 61210009, 61101221) and by National Key Technology R\&D Program (grant 2012BAI34B02).

\section{References}

[1] R. Szeliski, Image alignment and stitching: A tutorial, Found. and Trends in Computer Graphics and Vis. 2 (2006) 1-104.

[2] N. Snavely, S. M. Seitz, R. Szeliski, Photo tourism: exploring photo collections in 3D, ACM Trans. Graphics. 25 (2006) 835-846.

[3] L. Lin, X. Liu, S. C. Zhu, Layered graph matching with composite cluster sampling, IEEE Trans. Pattern Analysis and Machine Intell. 32 (2010) $1426-1442$.

[4] H. Li, E. Kim, X. Huang, L. He, Object matching with a locally affineinvariant constraint, in: Proc. IEEE Conf. Computer Vis. and Pattern Recognit., 2010, pp. 1641-1648.

[5] D. G. Lowe, Distinctive image features from scale-invariant keypoints, Int. J. Computer Vis. 60 (2004) 91-110.

[6] X. Yang, C. K. Liu, Z. Y. Liu, H. Qiao, B. F. Wang, Z. D. Wang, A probabilistic spectral graph matching algorithm for robust correspondence between lunar surface images, in: Proc. World Congress on Intell. Control and Automat., 2014, pp. $385-390$.

[7] W. Lian, D. Zhang, Rotation-invariant nonrigid point set matching in cluttered scenes, IEEE Trans. Image Process. 21 (2012) 2786-2797.

[8] B. Fan, F. Wu, Z. Hu, Towards reliable matching of images containing repetitive patterns, Pattern Recognit. Lett. 32 (2011) 1851-1859.

[9] M. Leordeanu, M. Hebert, A spectral technique for correspondence problems using pairwise constraints, in: Proc. IEEE Int. Conf. Computer Vis., 2005, pp. 1482-1489. 
[10] A. Noma, R. M. Cesar, Sparse representations for efficient shape matching, in: SIBGRAPI Conf. Graphics, Patterns and Images, 2010, pp. 186-192.

[11] A. C. Berg, T. L. Berg, J. Malik, Shape matching and object recognition using low distortion correspondences, in: IEEE Conf. Computer Vis. and Pattern Recognit., 2005, pp. 26-33.

[12] X. Yang, H. Qiao, Z. Y. Liu, Partial correspondence based on subgraph matching, Neurocomputing 122 (2013) 193-197.

[13] R. Zass, A. Shashua, Probabilistic graph and hypergraph matching, in: IEEE Conf. Computer Vis. and Pattern Recognit., 2008, pp. 1-8.

[14] O. Duchenne, F. Bach, I. S. Kweon, J. Ponce, A tensor-based algorithm for high-order graph matching, IEEE Trans. Pattern Analysis and Machine Intell. 33 (2011) 2383-2395.

[15] X. Yang, H. Qiao, Z. Y. Liu, Outlier robust point correspondence based on GNCCP, Pattern Recognit. Lett. 55 (2015) 8-14.

[16] H. Bay, T. Tuytelaars, L. Van Gool, SURF: Speeded up robust features, in: European Conf. Computer Vis., Springer, 2006, pp. 404-417.

[17] J. Matas, O. Chum, M. Urban, T. Pajdla, Robust wide-baseline stereo from maximally stable extremal regions, Image and Vis. Computing 22 (2004) 761-767.

[18] S. Leutenegger, M. Chli, R. Y. Siegwart, Brisk: Binary robust invariant scalable keypoints, in: Proc. IEEE Int. Conf. Computer Vis., 2011, pp. $2548-2555$.

[19] E. N. Mortensen, H. Deng, L. Shapiro, A SIFT descriptor with global context, in: IEEE Conf. Computer Vis. and Pattern Recognit., 2005, pp. $184-190$.

[20] L. Livi, A. Rizzi, The graph matching problem, Pattern Analysis and Applications 16 (2013) 253-283.

[21] P. Foggia, G. Percannella, M. Vento, Graph matching and learning in pattern recognition in the last 10 years, Int. J. Pattern Recognit. Artificial Intell. 28 (2014) 1450001-1 - 1450001-40. 
[22] S. Umeyama, An eigendecomposition approach to weighted graph matching problems, IEEE Trans. Pattern Analysis and Machine Intell. 10 (1988) 695-703.

[23] M. Cho, J. Lee, K. M. Lee, Reweighted random walks for graph matching, in: European Conf. Computer Vis., 2010, pp. 492-505.

[24] T. Cour, P. Srinivasan, J. Shi, Balanced graph matching, Advances in Neural Information Processing Systems 19 (2007) 313-320.

[25] S. Gold, A. Rangarajan, A graduated assignment algorithm for graph matching, IEEE Trans. Pattern Analysis and Machine Intell. 18 (1996) $377-388$.

[26] M. Zaslavskiy, F. Bach, J. P. Vert, A path following algorithm for the graph matching problem, IEEE Trans. Pattern Analysis and Machine Intell. 31 (2009) 2227-2242.

[27] Z. Y. Liu, H. Qiao, L. Xu, An extended path following algorithm for graph-matching problem, IEEE Trans. Pattern Analysis and Machine Intell. 34 (2012) 1451-1456.

[28] F. Zhou, F. De la Torre, Factorized graph matching, in: IEEE Conf. Computer Vis. and Pattern Recognit., 2012, pp. 127-134.

[29] B. Jiang, J. Tang, B. Luo, L. Lin, Robust feature point matching with sparse model, IEEE Trans. Image Processing 23 (2014) 5175-5186.

[30] Y. Zheng, D. Doermann, Robust point matching for nonrigid shapes by preserving local neighborhood structures, IEEE Trans. Pattern Analysis and Machine Intell. 28 (2006) 643-649.

[31] W. Aguilar, Y. Frauel, F. Escolano, M. E. Martinez Perez, A. EspinosaRomero, M. A. Lozano, A robust graph transformation matching for non-rigid registration, Image and Vis. Computing 27 (2009) 897-910.

[32] Z. Liu, J. An, Y. Jing, A simple and robust feature point matching algorithm based on restricted spatial order constraints for aerial image registration, IEEE Trans. Geoscience and Remote Sensing 50 (2012) $514-527$. 
[33] S. T. Roweis, L. K. Saul, Nonlinear dimensionality reduction by locally linear embedding, Science 290 (2000) 2323-2326.

[34] Z. Y. Liu, H. Qiao, GNCCP - graduated nonconvexity and concavity procedure, IEEE Trans. Pattern Analysis and Machine Intell. 36 (2014) $1258-1267$.

[35] X. Yang, H. Qiao, Z. Y. Liu, A weighted common subgraph matching algorithm, IEEE Trans. Neural Networks and Learning Systems, accepted and to appear. arXiv:1411.0763.

[36] M. Frank, P. Wolfe, An algorithm for quadratic programming, Nav. Res. Logist. Q. 3 (1956) 95-110.

[37] N. Japkowicz, M. Shah, Evaluating learning algorithms: a classification perspective, Cambridge University Press, 2011.

[38] M. Leordeanu, R. Sukthankar, M. Hebert, Unsupervised learning for graph matching, Int. J. Computer Vis. 96 (2012) 28-45.

[39] E. S. Ng, N. G. Kingsbury, Matching of interest point groups with pairwise spatial constraints, in: IEEE Conf. Image Processing, 2010, pp. 2693-2696.

[40] M. Cho, J. Lee, K. M. Lee, Feature correspondence and deformable object matching via agglomerative correspondence clustering, in: Proc. IEEE Int. Conf. Computer Vis., 2009, pp. 1280-1287. 
Yu-Ren Zhang received the bachelor degree from Beijing Jiaotong University, Beijing, China, in 2010. He is currently working toward the $\mathrm{PhD}$ degree in Institute of Automation, Chinese Academy of Sciences, Beijing China. His research interests include machine learning and graph matching.

Xu Yang is an assistant professor at the State Key Laboratory of Management and Control for Complex Systems, Institute of Automation, Chinese Academy of Sciences, Beijing, China. His research interests include computer vision, graph algorithms, and robotics.

Hong Qiao is a professor at the State Key Laboratory of Management and Control for Complex Systems, Institute of Automation, Chinese Academy of Sciences, Beijing, China. Her research interests include robotics, machine learning, and computer vision.

Zhi-Yong Liu is an associate professor at the State Key Laboratory of Management and Control for Complex Systems, Institute of Automation, Chinese Academy of Sciences, Beijing, China. His research interests include machine learning, pattern recognition, computer vision, and bioinformatics.

Chuan-Kai Liu received the Phd degree from Institute of Automation, Chinese Academy of Sciences, Beijing, China. He is currently working with the Beijing Aerospace Flight Control Center, Beijing, China . His current research interests include manipulation planning, vision-guided robotics, multiagent systems, high-precision assembly, intelligence fusion, and virtual reality. 


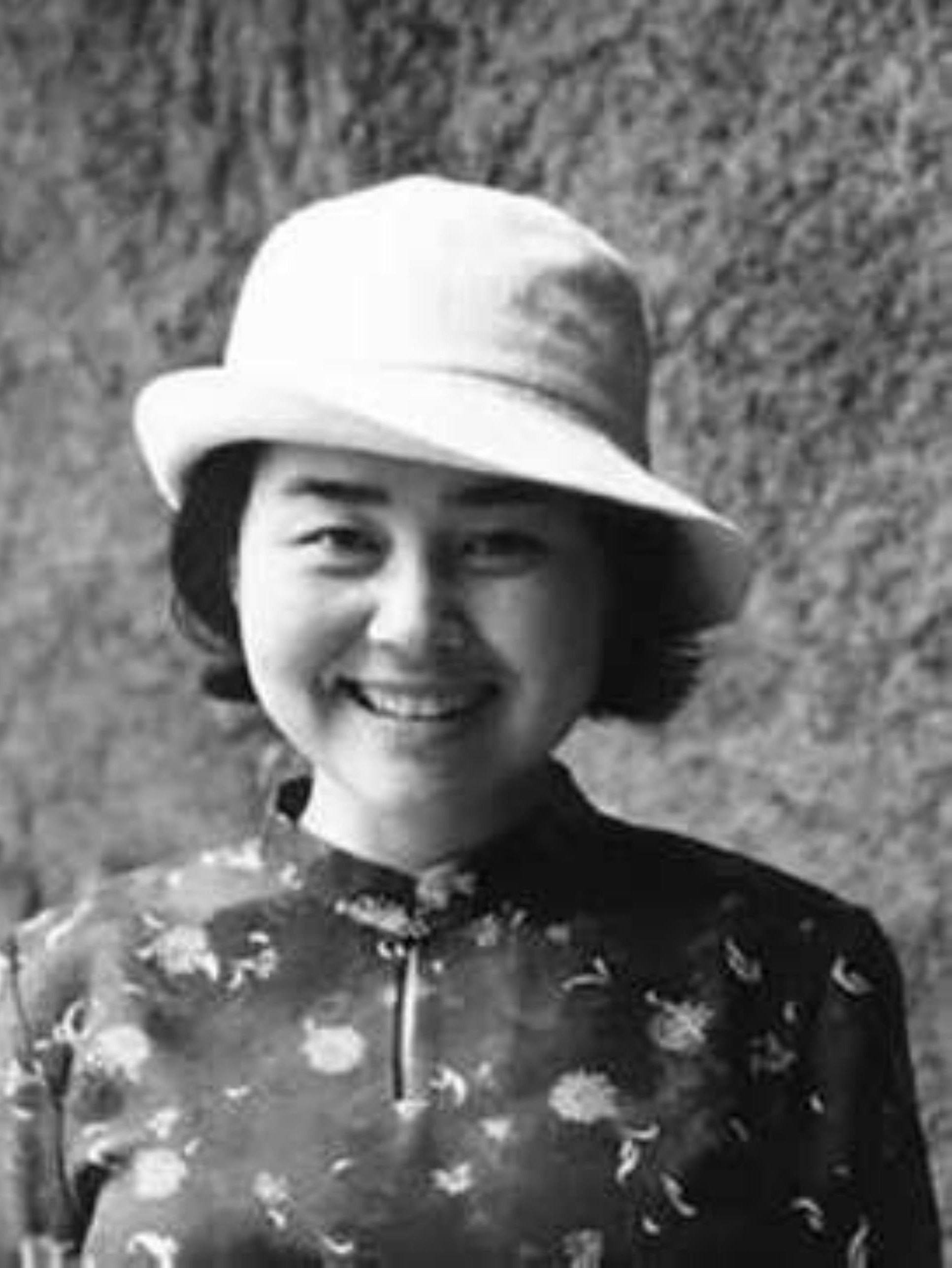


${ }^{*}$ Photo of the author(s)

Click here to download Photo of the author(s): Zhi-Yong Liu.eps

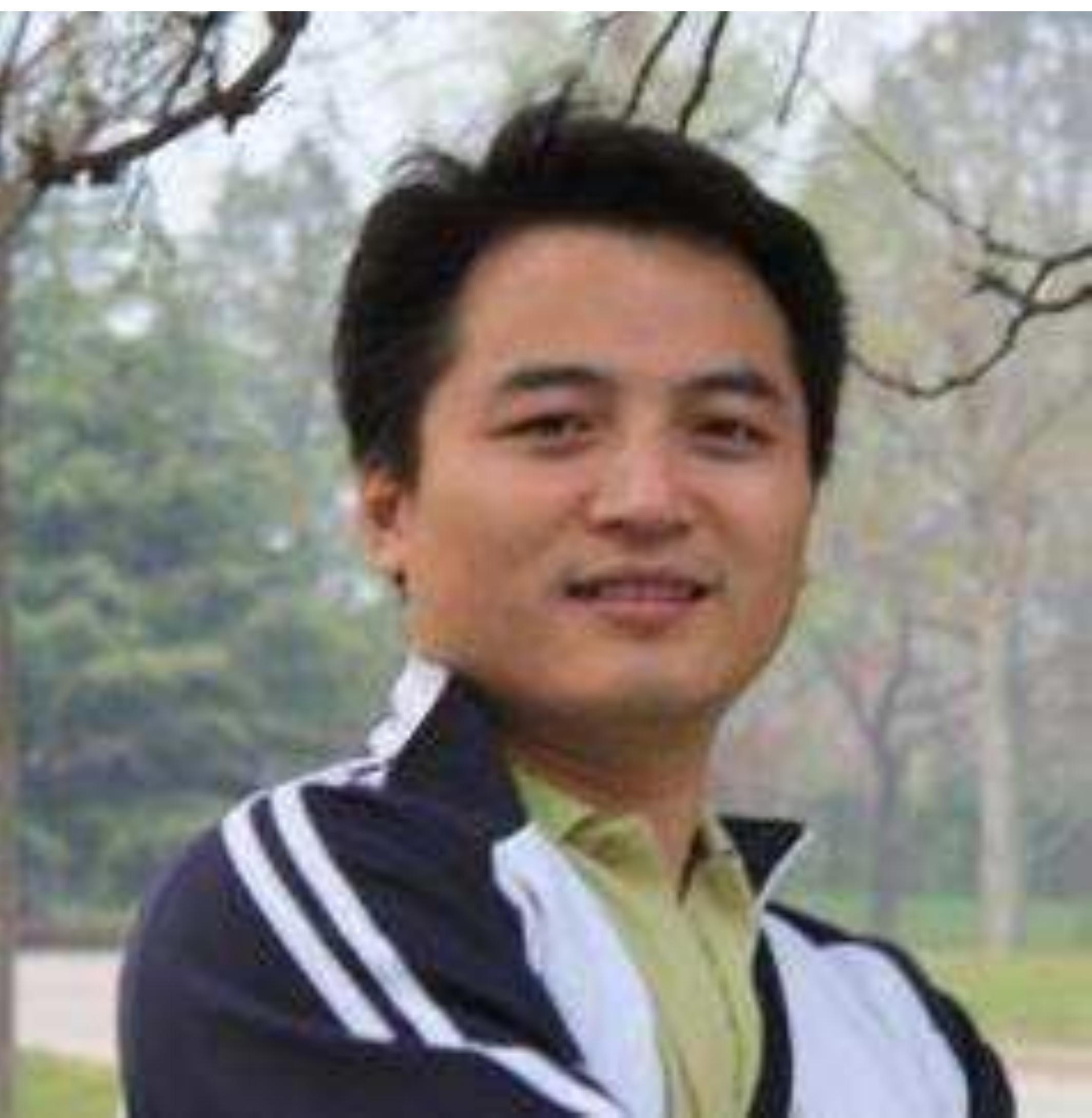


${ }^{*}$ Photo of the author(s)

Click here to download Photo of the author(s): yurenzhang.eps

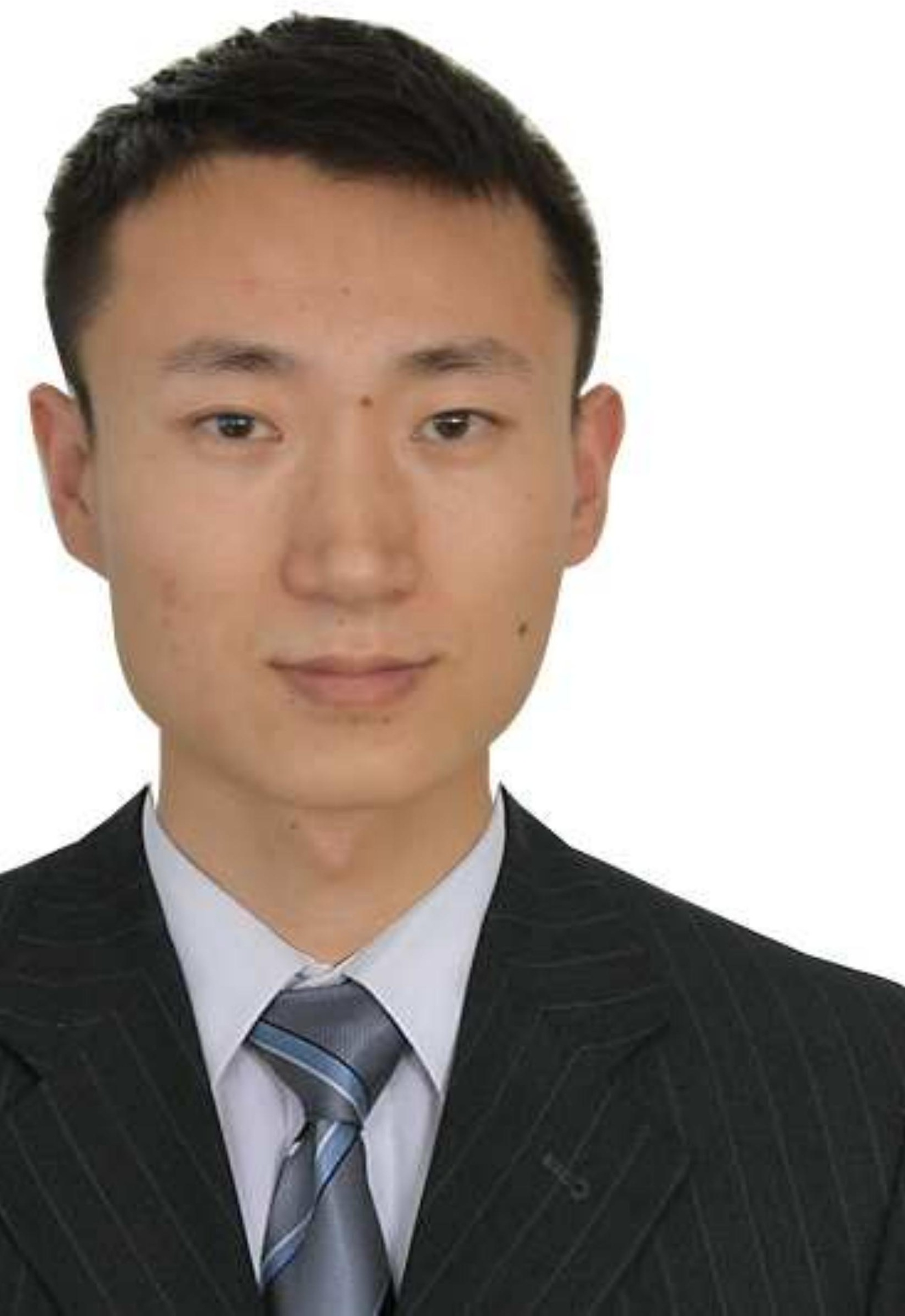


${ }^{*}$ Photo of the author(s)

Click here to download Photo of the author(s): Xu Yang.eps
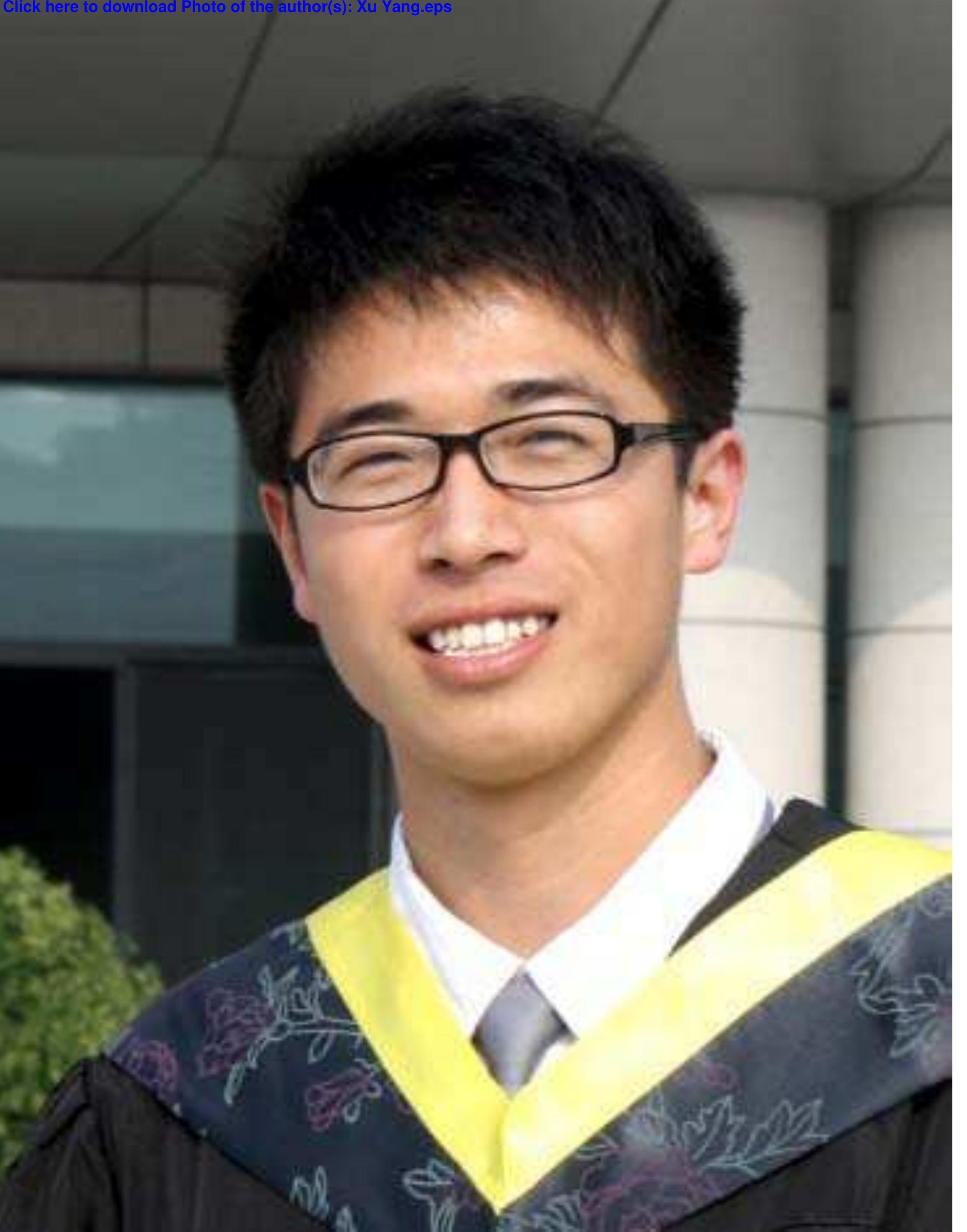
${ }^{*}$ Photo of the author(s)

Click here to download Photo of the author(s): chuankailiu.eps

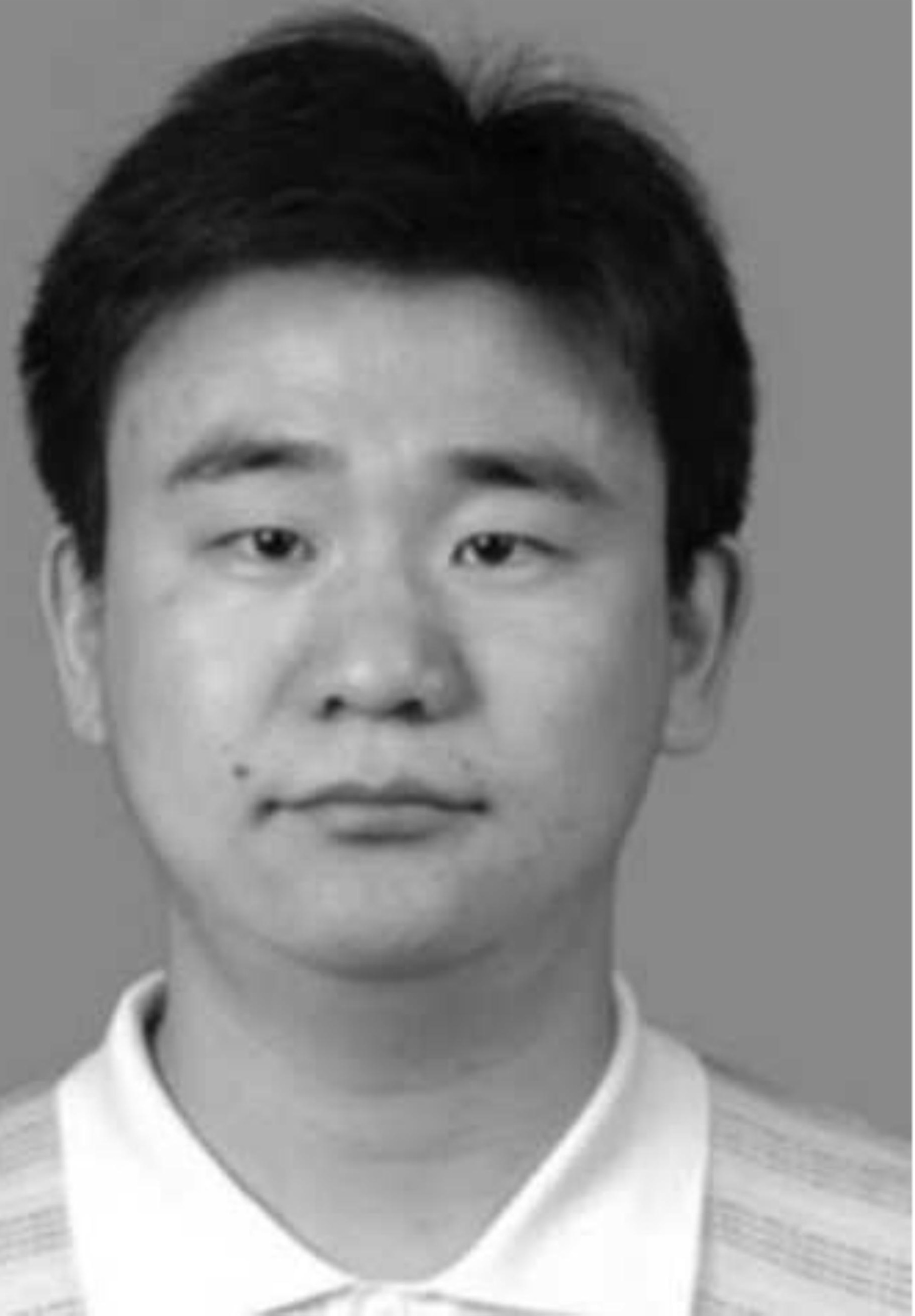

